For Internal Distribution Only

Accelerator Division

Alternating Gradient Synchrotron Department

BROOKHAVEN NATIONAL LABORATORY

Upton, New York 11973

Accelerator Division

Technical Note

AGS/AD/Tech. Note No. 427

NOTES ON COUPLED MOTION IN A LINEAR PERIODIC

LATTICE and APPLICATIONS TO BOOSTER INJECTION

C.J. Gardner

February 22, 1996 


\title{
Notes on Coupled Motion in a Linear Periodic Lattice and Applications to Booster Injection
}

\author{
C. J. Gardner
}

\section{Introduction}

For the past four years, coupling, introduced by skew quadrupoles, has been used during the injection of heavy ions in the Booster to enhance the efficiency of the injection process. The use of coupling was first proposed by Roser [1] who showed by modeling the process that the enhancement was actually quite significant. A coupled injection scheme was then developed for the Booster using existing skew quadrupoles and this has been used ever since for the injection of heavy ions. Although the scheme prescribes specific values for the tunes and skew quadrupole currents, the actual values are determined empirically using the prescribed values as a general guide. The purpose of the following report is to establish some additional guidelines to aid in the tuning of the coupled injection parameters. Sections $2-11$ are essentially a set of notes on coupled motion in a linear periodic lattice which are then applied in sections $12-17$ to the injection of heavy ions in the Booster.

\section{The Beam Ellipsoid}

Let $x_{0}, x_{0}^{\prime}, y_{0}, y_{0}^{\prime}$ be the horizontal and vertical positions and angles of a beam particle with respect to the reference trajectory at a point $s_{0}$ along the trajectory. Then the positions, $x$ and $y$, and angles, $x^{\prime}$ and $y^{\prime}$, of the particle at the point $s$ along the trajectory are given by

$$
\mathbf{Z}=\mathbf{T} \mathbf{Z}_{0}
$$


where

$$
\mathbf{Z}=\left(\begin{array}{c}
Z_{1} \\
Z_{2} \\
Z_{3} \\
Z_{4}
\end{array}\right)=\left(\begin{array}{l}
x \\
x^{\prime} \\
y \\
y^{\prime}
\end{array}\right), \quad \mathbf{z}_{0}=\left(\begin{array}{c}
x_{0} \\
x_{0}^{\prime} \\
y_{0} \\
y_{0}^{\prime}
\end{array}\right)
$$

and $\mathbf{T}$ is the four-by-four transfer matrix between $s_{0}$ and $s$. The matrix $\mathbf{T}$ is symplectic and has unit determinant.

Now suppose we define an ellipsoid at $s_{0}$ by

$$
\mathbf{Z}_{0}^{\dagger} \mathbf{E}_{0}^{-1} \mathbf{Z}_{0}=\epsilon
$$

where $\mathbf{E}_{0}^{-1}$ is a four-by-four real, symmetric, positive definite matrix with unit determinant. (We use a $\dagger$ to denote the transpose of a vector or matrix.) Then it is easy to show that the ellipsoid at $s_{0}$ is transformed into another ellipsoid at $s$. Using $\mathbf{Z}_{0}=T^{-1} \mathbf{Z}$ in (3) we find

$$
\mathbf{Z}^{\dagger} \mathbf{E}^{-1} \mathbf{Z}=\epsilon
$$

where

$$
\mathbf{E}=\mathbf{T} \mathbf{E}_{0} \mathbf{T}^{\dagger}
$$

Equation (4) defines an ellipsoid provided the matrix $\mathbf{E}^{-1}$ is symmetric and positive definite. Since $\mathbf{E}_{0}$ is symmetric $\left(\mathbf{E}_{0}^{\dagger}=\mathbf{E}_{0}\right)$ we have

$$
\mathbf{E}^{\dagger}=\mathbf{T} \mathbf{E}_{0}^{\dagger} \mathbf{T}^{\dagger}=\mathbf{T} \mathbf{E}_{0} \mathbf{T}^{\dagger}=\mathbf{E}
$$

and $\mathbf{E}^{-1}$ is therefore symmetric. Now a real and symmetric matrix, $\mathbf{A}$, is positive definite if the quadratic form $\mathbf{Z}^{\dagger} \mathbf{A Z}>\mathbf{0}$ for every $\mathbf{Z} \neq \mathbf{0}$. To show that $\mathbf{E}^{-1}$ is positive definite, consider

$$
\mathbf{Z}^{\dagger} \mathbf{E}^{-1} \mathbf{Z}=\mathbf{Z}_{0}^{\dagger} \mathbf{T}^{\dagger} \mathbf{E}^{-1} \mathbf{T} \mathbf{Z}_{0}=\mathbf{Z}_{0}^{\dagger} \mathbf{E}_{0}^{-1} \mathbf{Z}_{0}
$$

Since $\mathbf{Z}_{0}^{\dagger} \mathbf{E}_{0}^{-1} \mathbf{Z}_{0}>\mathbf{0}$ for all $\mathbf{Z}_{0} \neq \mathbf{0}$, and since $\mathbf{Z}=\mathbf{0}$ if and only if $\mathbf{Z}_{0}=\mathbf{0}$, it follows that $\mathbf{Z}^{\dagger} \mathbf{E}^{-1} \mathbf{Z}>0$ for all $\mathbf{Z} \neq \mathbf{0}$. Therefore $\mathbf{E}^{-1}$ is positive definite, and the ellipsoid defined by $\mathbf{E}_{0}$ is transformed into another ellipsoid defined by $\mathbf{E}$ in going from $s_{0}$ to $s$. We note that since $|\mathbf{T}|=1$,

$$
|\mathbf{E}|=|\mathbf{T}|\left|\mathbf{E}_{0}\right|\left|\mathbf{T}^{\dagger}\right|=\left|\mathbf{E}_{0}\right|,
$$

and the volume enclosed by the ellipsoid is therefore conserved. 
Now since $\mathbf{Z}^{\dagger} \mathbf{E}^{-1} \mathbf{Z}<\epsilon$ if and only if $\mathbf{Z}_{0}^{\dagger} \mathbf{E}_{0}^{-1} \mathbf{Z}_{0}<\epsilon$, we see that any particle inside (outside) the ellipsoid (3) at $s_{0}$ will be inside (outside) the ellipsoid (4) at $s$. Thus if we define the beam ellipsoid to be the smallest ellipsoid which contains the beam particles at $s_{0}$, then the evolution of this ellipsoid provides a convenient way of keeping track of the motion of the beam without having to track each particle individually. The ellipsoid is also useful for characterizing the motion of a single particle since any particle on the surface of the ellipsoid at $s_{0}$ will also be on the surface of the ellipsoid at $s$. In particular, if $\mathbf{T}$ is the transfer matrix for one period of a periodic lattice and $\mathbf{E}$ is an ellipsoid matrix which satisfies $\mathbf{E}=\mathbf{T E T}^{\dagger}$, then a given particle will always be on the surface of the ellipsoid $\mathbf{Z}^{\dagger} \mathbf{E}^{-1} \mathbf{Z}=\epsilon$ upon traversal of each period of the lattice.

\section{Maximum Extent in each Dimension}

Let us now determine the maximum extent, in each dimension, of the ellipsoid defined by (4). Since the matrix, $\mathbf{E}$, is real and symmetric there exists an orthogonal transformation, $\mathbf{O O}^{\dagger}=\mathbf{I}$, which diagonalizes $\mathbf{E}$. Thus

$$
\mathbf{O E O}^{\dagger}=\mathbf{e}=\left(\begin{array}{llll}
e_{1} & 0 & 0 & 0 \\
0 & e_{2} & 0 & 0 \\
0 & 0 & e_{3} & 0 \\
0 & 0 & 0 & e_{4}
\end{array}\right)
$$

and

$$
\mathbf{E}=\mathbf{O}^{\dagger} \mathbf{e O}, \quad \mathbf{E}^{-1}=\mathbf{O}^{\dagger} \mathbf{e}^{-1} \mathbf{O} .
$$

Since $\mathbf{E}$ is positive definite, its eigenvalues, the matrix elements $e_{i}$, are all positive, and it follows that

$$
E_{i i}=\sum_{j, k} O_{j i} e_{j k} O_{k i}=\sum_{k}\left(O_{k i}\right)^{2} e_{k}>0,
$$

i.e. the diagonal elements of $\mathbf{E}$ are all positive. Now let

$$
\mathbf{Y}=\mathbf{O Z}, \quad \mathbf{Z}=\mathbf{O}^{\dagger} \mathbf{Y}
$$

Then we have

$$
\epsilon=\mathbf{Z}^{\dagger} \mathbf{E}^{-1} \mathbf{Z}=\mathbf{Y}^{\dagger} \mathbf{O} \mathbf{E}^{-1} \mathbf{O}^{\dagger} \mathbf{Y}=\mathbf{Y}^{\dagger} \mathbf{e}^{-1} \mathbf{Y}
$$


and the equation of the ellipsoid in the transformed coordinates, $Y_{i}$, is

$$
\epsilon=\sum_{i} Y_{i}^{2} / e_{i}
$$

It follows that the maximum extent of each coordinate $Y_{i}$ is given by

$$
Y_{i}^{2} \leq \epsilon e_{i} .
$$

To obtain the maximum extent of the coordinate $Z_{i}$, we write

$$
Z_{i}=\sum_{j} O_{j i} Y_{j}=\sum_{j}\left(\sqrt{e_{j}} O_{j i}\right)\left(Y_{j} / \sqrt{e_{j}}\right) .
$$

Then making use of the Schwarz inequality [2] we have

$$
Z_{i}^{2} \leq \sum_{j}\left(O_{j i} \sqrt{e_{j}}\right)^{2} \sum_{j}\left(Y_{j} / \sqrt{e_{j}}\right)^{2},
$$

and using (11) and (14) in (17) we have

$$
Z_{i}^{2} \leq \epsilon E_{i i} \text {. }
$$

Thus the diagonal elements of $\mathbf{E}$ give the maximum extent in each dimension of the ellipsoid defined by $\mathbf{Z}^{\dagger} \mathbf{E}^{-1} \mathbf{Z}=\epsilon$.

The equality in (17) and (18) holds if and only if $Y_{j} / \sqrt{e_{j}}=\lambda O_{j i} \sqrt{e_{j}}$, which is true if and only if

$$
Y_{j}=\lambda O_{j i} e_{j} .
$$

Using this equation in (14) we find

$$
\epsilon=\lambda^{2} \sum_{j}\left(O_{j i}\right)^{2} e_{j}=\lambda^{2} E_{i i}
$$

and therefore

$$
\lambda^{2}=\epsilon / E_{i i} .
$$

Then using (12), (19) and (10) we find

$$
Z_{k}=\sum_{j} O_{j k} Y_{j}=\lambda \sum_{j} O_{j k} e_{j} O_{j i}=\lambda E_{k i}
$$

and therefore

$$
Z_{i}=\lambda E_{i i}= \pm \sqrt{\epsilon E_{i i}}, \quad Z_{k}=\left(E_{k i} / E_{i i}\right) Z_{i} .
$$

Thus when $Z_{i}^{2}=\epsilon E_{i i}$ the other components of $\mathbf{Z}$ are given by the second of equations (23). 


\section{Projections}

We can learn more about the size and shape of the ellipsoid by examining its projections onto the $\left(x, x^{\prime}\right),\left(y, y^{\prime}\right)$ and $(x, y)$ planes. To obtain the projections we partition the matricies in equations (1) and (5) into two-by-two matricies. Introducing the notation

$$
\mathbf{E}_{0}=\left(\begin{array}{ll}
\mathbf{F}_{0} & \mathbf{C}_{0} \\
\mathbf{C}_{0}^{\dagger} & \mathbf{G}_{0}
\end{array}\right), \quad \mathbf{E}=\left(\begin{array}{ll}
\mathbf{F} & \mathbf{C} \\
\mathbf{C}^{\dagger} & \mathbf{G}
\end{array}\right), \quad \mathbf{T}=\left(\begin{array}{cc}
\mathbf{M} & \mathbf{n} \\
\mathbf{m} & \mathbf{N}
\end{array}\right)
$$

and

$$
\begin{gathered}
\mathbf{Z}_{0}=\left(\begin{array}{c}
\mathbf{X}_{0} \\
\mathbf{Y}_{0}
\end{array}\right), \quad \mathbf{X}_{0}=\left(\begin{array}{c}
x_{0} \\
x_{0}^{\prime}
\end{array}\right), \quad \mathbf{Y}_{0}=\left(\begin{array}{c}
y_{0} \\
y_{0}^{\prime}
\end{array}\right), \\
\mathbf{Z}=\left(\begin{array}{c}
\mathbf{X} \\
\mathbf{Y}
\end{array}\right), \quad \mathbf{X}=\left(\begin{array}{c}
x \\
x^{\prime}
\end{array}\right), \quad \mathbf{Y}=\left(\begin{array}{c}
y \\
y^{\prime}
\end{array}\right)
\end{gathered}
$$

where $\mathbf{F}_{0}, \mathbf{G}_{0}, \mathbf{C}_{0}, \mathbf{F}, \mathbf{G}, \mathbf{C}, \mathbf{M}, \mathbf{N}, \mathbf{m}, \mathbf{n}$ are two-by-two matricies, the equation $\mathbf{E}=\mathbf{T} \mathbf{E}_{0} \mathbf{T}^{\dagger}$ becomes

$$
\begin{aligned}
\mathbf{F} & =\mathbf{M} \mathbf{F}_{0} \mathbf{M}^{\dagger}+\mathbf{n} \mathbf{G}_{0} \mathbf{n}^{\dagger}+\mathbf{n} \mathbf{C}_{0}^{\dagger} \mathbf{M}^{\dagger}+\mathbf{M} \mathbf{C}_{0} \mathbf{n}^{\dagger} \\
\mathbf{G} & =\mathbf{N} \mathbf{G}_{0} \mathbf{N}^{\dagger}+\mathbf{m} \mathbf{F}_{0} \mathbf{m}^{\dagger}+\mathbf{N} \mathbf{C}_{0}^{\dagger} \mathbf{m}^{\dagger}+\mathbf{m} \mathbf{C}_{0} \mathbf{N}^{\dagger} \\
\mathbf{C} & =\mathbf{M} \mathbf{C}_{0} \mathbf{N}^{\dagger}+\mathbf{n} \mathbf{C}_{0}^{\dagger} \mathbf{m}^{\dagger}+\mathbf{M} \mathbf{F}_{0} \mathbf{m}^{\dagger}+\mathbf{n} \mathbf{G}_{0} \mathbf{N}^{\dagger}
\end{aligned}
$$

and $\mathbf{Z}=\mathbf{T} \mathbf{Z}_{0}$ becomes

$$
\mathbf{X}=\mathbf{M X _ { 0 }}+\mathbf{n} \mathbf{Y}_{0}, \quad \mathbf{Y}=\mathbf{m} \mathbf{X}_{0}+\mathbf{N} \mathbf{Y}_{0}
$$

Now consider the ellipsoid $\mathbf{Z}_{0}^{\dagger} \mathbf{E}_{0}^{-1} \mathbf{Z}_{0}=\epsilon$. To find the projection onto the $x_{0}, x_{0}^{\prime}$ plane we seek a transformation, $\mathbf{T}$, from coordinates given by $\mathbf{X}_{0}$, $\mathbf{Y}_{0}$ to new coordinates given by $\mathbf{X}, \mathbf{Y}$ such that $\mathbf{X}=\mathbf{X}_{\mathbf{0}}$ and the equation for the transformed ellipsoid is of the form

$$
\mathbf{X}^{\dagger} \mathbf{F}^{-1} \mathbf{X}+\mathbf{Y}^{\dagger} \mathbf{G}^{-1} \mathbf{Y}=\epsilon
$$

where $\mathbf{F}^{-1}$ and $\mathbf{G}^{-1}$ are positive definite. We obtain such a transformation if we choose

$$
\mathbf{M}=\mathbf{N}=\mathbf{I}, \quad \mathbf{n}=\mathbf{0}, \quad \mathbf{m}=-\mathbf{C}_{0}^{\dagger} \mathbf{F}_{0}^{-1}
$$


Then we have

$$
\mathbf{T}=\left(\begin{array}{cc}
\mathbf{I} & \mathbf{0} \\
\mathbf{m} & \mathbf{I}
\end{array}\right), \quad \mathbf{T}^{-1}=\left(\begin{array}{cc}
\mathbf{I} & \mathbf{0} \\
-\mathbf{m} & \mathbf{I}
\end{array}\right)
$$

and the equation $\mathbf{E}=\mathbf{T} \mathbf{E}_{0} \mathbf{T}^{\dagger}$ becomes

$$
\mathbf{F}=\mathbf{F}_{0}, \quad \mathbf{C}=0, \quad \mathbf{G}=\mathbf{G}_{0}-\mathbf{C}_{0}^{\dagger} \mathbf{F}_{0}^{-1} \mathbf{C}_{0}
$$

Thus

$$
\mathbf{E}^{-1}=\left(\begin{array}{ll}
\mathbf{F}^{-1} & \mathbf{0} \\
\mathbf{0} & \mathbf{G}^{-1}
\end{array}\right)
$$

and the equation for the transformed ellipsoid is

$$
\mathbf{Z}^{\dagger} \mathbf{E}^{-1} \mathbf{Z}=\mathbf{X}^{\dagger} \mathbf{F}^{-1} \mathbf{X}+\mathbf{Y}^{\dagger} \mathbf{G}^{-1} \mathbf{Y}=\epsilon .
$$

where

$$
\mathbf{X}=\mathbf{X}_{0}, \quad \mathbf{Y}=\mathbf{Y}_{0}-\mathbf{C}_{0}^{\dagger} \mathbf{F}_{0}^{-1} \mathbf{X}_{0}, \quad \mathbf{F}=\mathbf{F}_{0} .
$$

Now since $\mathbf{E}_{0}^{-1}$ is positive definite and since $\mathbf{T}^{-1}$ exists, the matrix $\mathbf{E}^{-1}$ is positive definite. The quadratic forms $\mathbf{X}^{\dagger} \mathbf{F}^{-1} \mathbf{X}$ and $\mathbf{Y}^{\dagger} \mathbf{G}^{-1} \mathbf{Y}$ are therefore positive definite and it follows that

$$
\mathbf{X}_{0}^{\dagger} \mathbf{F}_{0}^{-1} \mathbf{X}_{0} \leq \epsilon
$$

Defining

$$
\mathbf{f}_{0}=\mathbf{F}_{0} / D, \quad D=\left|\mathbf{F}_{0}\right|^{1 / 2}
$$

we obtain

$$
\mathbf{X}_{0}^{\dagger} \mathbf{f}_{0}^{-1} \mathbf{X}_{0} \leq \epsilon D, \quad\left|\mathbf{f}_{0}\right|=1
$$

which defines an elliptical region of area $\pi \epsilon D$. This region is the projection of the ellipsoid $\mathbf{Z}_{0}^{\dagger} \mathbf{E}_{0}^{-1} \mathbf{Z}_{0}=\epsilon$ onto the $x_{0}, x_{0}^{\prime}$ plane. The boundry of this region is given by

$$
\mathbf{X}_{0}^{\dagger} \mathbf{F}_{0}^{-1} \mathbf{X}_{0}=\epsilon
$$

For these points we have

$$
\mathbf{Y}^{\dagger} \mathbf{G}^{-1} \mathbf{Y}=0, \quad \mathbf{Y}=\mathbf{0},
$$

and therefore

$$
\mathbf{Y}_{0}=\mathbf{C}_{0}^{\dagger} \mathbf{F}_{0}^{-1} \mathbf{X}_{0}
$$


Thus equations (41) and (43) give the points on the surface of the ellipsoid which project onto the boundry of the $x_{0}, x_{0}^{\prime}$ projection.

Generalizing the argument given above we find that the projection of the ellipsoid, $\mathbf{Z}^{\dagger} \mathbf{E}^{-1} \mathbf{Z}=\epsilon$, on the $Z_{m}, Z_{n}$ plane is the elliptical region.

$$
\mathbf{X}^{\dagger} \mathbf{f}^{-1} \mathbf{X} \leq \epsilon D
$$

where

$$
\mathbf{X}=\left(\begin{array}{c}
Z_{m} \\
Z_{n}
\end{array}\right), \quad D=\left(E_{m m} E_{n n}-E_{m n}^{2}\right)^{1 / 2},
$$

and the matrix elements of $f$ are

$$
f_{11}=E_{m m} / D, \quad f_{22}=E_{n n} / D, \quad f_{12}=f_{21}=E_{m n} / D
$$

\section{The One-Turn Transfer Matrix}

For the remainder of these notes we will use $\mathbf{T}$ to denote the transfer matrix for one turn around a ring or for a pass through one period of a periodic lattice. In this section we examine the symplectic nature of $\mathbf{T}$. By definition, a four-by-four matrix $\mathbf{T}$ is symplectic if

$$
\mathbf{T}^{\dagger} \mathbf{S T}=\mathbf{S}
$$

where

$$
\mathbf{S}=\left(\begin{array}{rrrr}
0 & 1 & 0 & 0 \\
-1 & 0 & 0 & 0 \\
0 & 0 & 0 & 1 \\
0 & 0 & -1 & 0
\end{array}\right), \quad-\mathbf{S}^{2}=\mathbf{I}=\left(\begin{array}{llll}
1 & 0 & 0 & 0 \\
0 & 1 & 0 & 0 \\
0 & 0 & 1 & 0 \\
0 & 0 & 0 & 1
\end{array}\right)
$$

Taking the inverse of both sides of (47) we obtain $\mathbf{T}^{-1} \mathbf{S}\left(\mathbf{T}^{\dagger}\right)^{-1}=\mathbf{S}$ and therefore

$$
\mathbf{S}=\mathbf{T S} \mathbf{T}^{\dagger}
$$

which is an equivalent form of the symplectic condition. Now, following Courant and Snyder [3], we define the symplectic conjugate of a two-by-two or four-by-four matrix $\mathbf{A}$ to be

$$
\overline{\mathbf{A}}=-\mathbf{S A}^{\dagger} \mathbf{S},
$$


where $\mathbf{S}$ is given by (48) for the case of four-by-four matricies. For two-by-two matricies we have

$$
\mathbf{A}=\left(\begin{array}{ll}
A_{11} & A_{12} \\
A_{21} & A_{22}
\end{array}\right), \quad \mathbf{S}=\left(\begin{array}{rr}
0 & 1 \\
-1 & 0
\end{array}\right), \quad \overline{\mathbf{A}}=\left(\begin{array}{rr}
A_{22} & -A_{12} \\
-A_{21} & A_{11}
\end{array}\right),
$$

and it follows that (for two-by-two matricies)

$$
\begin{gathered}
\mathbf{A} \overline{\mathbf{A}}=\overline{\mathbf{A}} \mathbf{A}=\left(A_{11} A_{22}-A_{12} A_{21}\right) \mathbf{I}=|\mathbf{A}| \mathbf{I} \\
\mathbf{A}+\overline{\mathbf{A}}=\left(A_{11}+A_{22}\right) \mathbf{I}=\operatorname{Tr}(\mathbf{A}) \mathbf{I} .
\end{gathered}
$$

Using (47) and (49) we have

$$
\overline{\mathbf{T}} \mathbf{T}=-\mathbf{S} \mathbf{T}^{\dagger} \mathbf{S T}=-\mathbf{S}^{2}=\mathbf{I}, \quad \mathbf{T} \overline{\mathbf{T}}=-\mathbf{T S} \mathbf{T}^{\dagger} \mathbf{S}=-\mathbf{S}^{2}=\mathbf{I}
$$

and therefore $\overline{\mathbf{T}}=\mathbf{T}^{-1}$ if $\mathbf{T}$ is symplectic. Then writing

$$
\mathbf{T}=\left(\begin{array}{cc}
\mathbf{M} & \mathbf{n} \\
\mathbf{m} & \mathbf{N}
\end{array}\right)
$$

where $\mathbf{M}, \mathbf{N}, \mathbf{m}, \mathbf{n}$ are two-by-two matricies, we have

$$
\mathbf{T} \overline{\mathbf{T}}=\left(\begin{array}{rr}
\mathbf{M} & \mathbf{n} \\
\mathbf{m} & \mathbf{N}
\end{array}\right)\left(\begin{array}{rr}
\overline{\mathbf{M}} & \overline{\mathbf{m}} \\
\overline{\mathbf{n}} & \overline{\mathbf{N}}
\end{array}\right)=\left(\begin{array}{cc}
\mathbf{M} \overline{\mathbf{M}}+\mathbf{n} \overline{\mathbf{n}} & \mathbf{M} \overline{\mathbf{m}}+\mathbf{n} \overline{\mathbf{N}} \\
\mathbf{m} \overline{\mathbf{M}}+\mathbf{N} \overline{\mathbf{n}} & \mathbf{m} \overline{\mathbf{m}}+\mathbf{N} \overline{\mathbf{N}}
\end{array}\right)
$$

and

$$
\overline{\mathbf{T}} \mathbf{T}=\left(\begin{array}{cc}
\overline{\mathbf{M}} & \overline{\mathbf{m}} \\
\overline{\mathbf{n}} & \overline{\mathbf{N}}
\end{array}\right)\left(\begin{array}{cc}
\mathbf{M} & \mathbf{n} \\
\mathbf{m} & \mathbf{N}
\end{array}\right)=\left(\begin{array}{cc}
\overline{\mathbf{M}} \mathbf{M}+\overline{\mathbf{m}} \mathbf{m} & \overline{\mathbf{M}} \mathbf{n}+\overline{\mathbf{m}} \mathbf{N} \\
\overline{\mathbf{n}} \mathbf{M}+\overline{\mathbf{N}} \mathbf{m} & \overline{\mathbf{n}} \mathbf{n}+\overline{\mathbf{N}} \mathbf{N}
\end{array}\right)
$$

and comparing (57) with $\overline{\mathbf{T}} \mathbf{T}=\mathbf{I}$ we find

$$
|\mathbf{M}|+|\mathbf{m}|=1, \quad|\mathbf{N}|+|\mathbf{n}|=1, \quad \overline{\mathbf{M}} \mathbf{n}+\overline{\mathbf{m}} \mathbf{N}=\mathbf{0} .
$$

Thus the symplectic condition imposes a total of 6 constraints on the 16 matrix elements of $T$, and $T$ is therefore specified by 10 independent parameters. Comparing (56) with $\mathbf{T} \overline{\mathbf{T}}=\mathbf{I}$ we also obtain

$$
|\mathbf{M}|+|\mathbf{n}|=1, \quad|\mathbf{N}|+|\mathbf{m}|=1, \quad \mathbf{M} \overline{\mathbf{m}}+\mathbf{n} \overline{\mathbf{N}}=\mathbf{0}
$$

which are equivalent to (58). 


\section{Reduction to Block-Diagonal Form}

It has been shown by Teng $[4,5]$ and more recently by Roser [6] that $\mathbf{T}$ can be expressed in the form

$$
\mathbf{T}=\mathbf{R} \mathbf{U} \mathbf{R}^{-1}, \quad \mathbf{U}=\left(\begin{array}{cc}
\mathbf{A} & \mathbf{0} \\
\mathbf{0} & \mathbf{B}
\end{array}\right)
$$

where $\mathbf{A}$ and $\mathbf{B}$ are two-by-two matricies and $\mathbf{U}$ and $\mathbf{R}$ are symplectic. We review here the treatment of Roser which overcomes some deficiencies of Teng's treatment.

Since $U$ is symplectic we must have $|\mathbf{A}|=|\mathbf{B}|=1$ and it follows that $U$ is specified by six independent parameters. The remaining four parameters needed to completely specify $\mathbf{T}$ are contained in the matrix $\mathbf{R}$. To express $\mathbf{R}$ in terms of four independent parameters we first choose

$$
R_{11}=R_{22}=R_{33}=R_{44}=d, \quad R_{12}=R_{21}=R_{34}=R_{43}=0 .
$$

Then it follows from the symplectic condition that $\mathbf{R}$ is of the form

$$
\mathbf{R}=\left(\begin{array}{cc}
d \mathbf{I} & \overline{\mathbf{W}} \\
-\mathbf{W} & d \mathbf{I}
\end{array}\right), \quad \mathbf{W}=\left(\begin{array}{ll}
W_{11} & W_{12} \\
W_{21} & W_{22}
\end{array}\right), \quad \mathbf{I}=\left(\begin{array}{cc}
1 & 0 \\
0 & 1
\end{array}\right)
$$

where

$$
d^{2}=1-|\mathbf{W}|, \quad d^{2} \mathbf{I}=\mathbf{I}-\mathbf{W} \overline{\mathbf{W}}
$$

Now writing

$$
\mathbf{T}=\mathbf{R U} \overline{\mathbf{R}}=\left(\begin{array}{cc}
d \mathbf{I} & \overline{\mathbf{W}} \\
-\mathbf{W} & d \mathbf{I}
\end{array}\right)\left(\begin{array}{cc}
\mathbf{A} & \mathbf{0} \\
\mathbf{0} & \mathbf{B}
\end{array}\right)\left(\begin{array}{cc}
d \mathbf{I} & -\overline{\mathbf{W}} \\
\mathbf{W} & d \mathbf{I}
\end{array}\right)
$$

we find

$$
\begin{array}{ll}
\mathbf{M}=d^{2} \mathbf{A}+\overline{\mathbf{W}} \mathbf{B W}, & \mathbf{n}=d \overline{\mathbf{W}} \mathbf{B}-d \mathbf{A} \overline{\mathbf{W}} \\
\mathbf{m}=d \mathbf{B W}-d \mathbf{W A}, & \mathbf{N}=d^{2} \mathbf{B}+\mathbf{W A} \overline{\mathbf{W}} .
\end{array}
$$

We see here that if $d=\mathbf{0}$ then $\mathbf{m}=\mathbf{n}=\mathbf{0}$ and $\mathbf{T}$ is in block-diagonal form. Since we are considering transfer matricies for which $\mathbf{m}$ and $\mathbf{n}$ are nonzero, we shall assume that $d \neq 0$. Now subtracting $\mathbf{N}$ from $\mathbf{M}$ we have

$$
\mathbf{M}-\mathbf{N}=d^{2}(\mathbf{A}-\mathbf{B})+\overline{\mathbf{W B}} \mathbf{B}-\mathbf{W A} \overline{\mathbf{W}}
$$


and taking the trace of this equation we obtain

$$
T=d^{2} U-|\mathbf{W}| U=\left(2 d^{2}-1\right) U
$$

where

$$
T=\operatorname{Tr}(\mathbf{M}-\mathbf{N}), \quad U=\operatorname{Tr}(\mathbf{A}-\mathbf{B}) .
$$

Thus we can write

$$
U+T=2 U d^{2}, \quad U-T=2 U\left(1-d^{2}\right), \quad U^{2}-T^{2}=4 d^{2} U^{2}\left(1-d^{2}\right) .
$$

Now adding $\mathbf{m}$ and $\overline{\mathbf{n}}$ we have

$$
\begin{aligned}
\mathbf{m}+\overline{\mathbf{n}} & =d \mathbf{B W}-d \mathbf{W A}+d \overline{\mathbf{B}} \mathbf{W}-d \mathbf{W} \overline{\mathbf{A}} \\
& =(\mathbf{B}+\overline{\mathbf{B}}) d \mathbf{W}-d \mathbf{W}(\mathbf{A}+\overline{\mathbf{A}})
\end{aligned}
$$

and using (53) we obtain

$$
\mathbf{m}+\overline{\mathbf{n}}=-d U \mathbf{W} .
$$

Multiplying this equation by $\mathbf{n}$ and using $|\mathbf{n}|=|\mathbf{m}|$, which follows from (58) and (59), we have

$$
-d U \mathbf{W n}=\mathbf{m n}+\overline{\mathbf{n}} \mathbf{n}=\mathbf{m} \mathbf{n}+\mathbf{m} \overline{\mathbf{m}}
$$

and

$$
-d U \mathbf{n} \mathbf{W}=\mathbf{n} \mathbf{m}+\mathbf{n} \overline{\mathbf{n}}=\mathbf{n} \mathbf{m}+\overline{\mathbf{m}} \mathbf{m} .
$$

We can also multiply the second of equations (65) by $W$ to obtain

$$
\mathbf{W n}=d(\mathbf{W} \overline{\mathbf{W}} \mathbf{B}-\mathbf{W A} \overline{\mathbf{W}}), \quad \mathbf{n} \mathbf{W}=d(\overline{\mathbf{W}} \mathbf{B} \mathbf{W}-\mathbf{A} \overline{\mathbf{W}} \mathbf{W})
$$

and using these equations in (73) and (74), we obtain

$$
\begin{aligned}
& d^{2} U(\mathbf{W A} \overline{\mathbf{W}}-\mathbf{W} \overline{\mathbf{W}} \mathbf{B})=\mathbf{m} \mathbf{n}+\mathbf{m} \overline{\mathbf{m}} \\
& d^{2} U(\mathbf{A} \overline{\mathbf{W}} \mathbf{W}-\overline{\mathbf{W}} \mathbf{B W})=\mathbf{n} \mathbf{m}+\overline{\mathbf{m}} \mathbf{m} .
\end{aligned}
$$

Then taking the trace of (77) we obtain

$$
d^{2} U^{2}|\mathbf{W}|=d^{2} U^{2}\left(1-d^{2}\right)=\operatorname{Tr}(\mathbf{m} \mathbf{n})+2|\mathbf{m}|
$$

and using this in the last of equations (70) we obtain

$$
U^{2}=T^{2}+4 \operatorname{Tr}(\mathbf{m n})+8|\mathbf{m}| \text {. }
$$


Thus we have obtained an expression for $U$ which contains only the matrix elements of $\mathbf{T}$. Now using (63), (65) and (66) we have

$$
\begin{aligned}
& \mathbf{M}-\mathbf{A}=\left(d^{2}-1\right) \mathbf{A}+\overline{\mathbf{W}} \mathbf{B} \mathbf{W}=\overline{\mathbf{W}} \mathbf{B} \mathbf{W}-\mathbf{A} \overline{\mathbf{W}} \mathbf{W}, \\
& \mathbf{N}-\mathbf{B}=\left(d^{2}-1\right) \mathbf{B}+\mathbf{W} \mathbf{A} \overline{\mathbf{W}}=\mathbf{W} \mathbf{A} \overline{\mathbf{W}}-\mathbf{W} \overline{\mathbf{W}} \mathbf{B},
\end{aligned}
$$

and therefore

$$
\begin{aligned}
& \mathbf{A}=\mathbf{M}+(\mathbf{A} \overline{\mathbf{W}} \mathbf{W}-\overline{\mathbf{W}} \mathbf{B} \mathbf{W}) \\
& \mathbf{B}=\mathbf{N}-(\mathbf{W} \mathbf{A} \overline{\mathbf{W}}-\mathbf{W} \overline{\mathbf{W}} \mathbf{B}) .
\end{aligned}
$$

Using (70), (76) and (77) we then have

$$
\begin{aligned}
& \mathbf{A}=\mathbf{M}+\frac{2}{U+T}(\mathbf{n m}+\overline{\mathbf{m}} \mathbf{m}) \\
& \mathbf{B}=\mathbf{N}-\frac{2}{U+T}(\mathbf{m} \mathbf{n}+\mathbf{m} \overline{\mathbf{m}}) .
\end{aligned}
$$

Thus if we are given the transfer matrix $\mathbf{T}$ we can obtain the matricies $\mathbf{R}$ and $\mathbf{U}$ by making the following sequence of evaluations: First we evaluate the right side of equation (79) to obtain $U^{2}$ which gives us the magnitude of $U$ but not its sign. To determine the sign, we substitute $U= \pm \sqrt{U^{2}}$ into the first of equations (70) and choose the sign which gives $d^{2}>0$. Having determined $U$ we can obtain $\mathbf{A}$ and $\mathbf{B}$ from (84) and (85). To obtain $\mathbf{W}$ we choose $d=+\sqrt{d^{2}}$ and substitute this along with $U$ into (72).

Now we define Courant-Snyder parameters such that

$$
\begin{aligned}
\cos \psi_{1}=\left(A_{11}+A_{22}\right) / 2, & \cos \psi_{2}=\left(B_{11}+B_{22}\right) / 2 \\
\alpha_{1} \sin \psi_{1}=\left(A_{11}-A_{22}\right) / 2, & \alpha_{2} \sin \psi_{2}=\left(B_{11}-B_{22}\right) / 2, \\
\beta_{1} \sin \psi_{1}=A_{12}, & \beta_{2} \sin \psi_{2}=B_{12} \\
\gamma_{1} \sin \psi_{1}=-A_{21}, & \gamma_{2} \sin \psi_{2}=-B_{21},
\end{aligned}
$$

where $\beta_{1}$ and $\beta_{2}$ are positive. Then $\mathbf{A}$ and $\mathbf{B}$ are of the form

$$
\mathbf{A}=\mathbf{I} \cos \psi_{1}+\mathbf{J}_{1} \sin \psi_{1}, \quad \mathbf{B}=\mathbf{I} \cos \psi_{2}+\mathbf{J}_{2} \sin \psi_{2}
$$

where

$$
\mathbf{I}=\left(\begin{array}{ll}
1 & 0 \\
0 & 1
\end{array}\right), \quad \mathbf{J}_{1}=\left(\begin{array}{cc}
\alpha_{1} & \beta_{1} \\
-\gamma_{1} & -\alpha_{1}
\end{array}\right), \quad \mathbf{J}_{2}=\left(\begin{array}{cc}
\alpha_{2} & \beta_{2} \\
-\gamma_{2} & -\alpha_{2}
\end{array}\right)
$$


and since $|\mathbf{A}|=|\mathbf{B}|=1$ we have

$$
\beta_{1} \gamma_{1}-\alpha_{1}^{2}=1, \quad \beta_{2} \gamma_{2}-\alpha_{2}^{2}=1
$$

Now since

$$
\mathbf{J}_{1}^{2}=-\mathbf{I}, \quad \mathbf{J}_{2}^{2}=-\mathbf{I}
$$

it follows that

$$
\mathbf{A}^{n}=\mathbf{I} \cos n \psi_{1}+\mathbf{J}_{1} \sin n \psi_{1}, \quad \mathbf{B}^{n}=\mathbf{I} \cos n \psi_{2}+\mathbf{J}_{2} \sin n \psi_{2},
$$

and using this in

$$
\mathbf{T}^{n}=\mathbf{R} \cdot \mathbf{U}^{n} \mathbf{R}^{-1}, \quad \mathbf{U}^{n}=\left(\begin{array}{cc}
\mathbf{A}^{n} & \mathbf{0} \\
\mathbf{0} & \mathbf{B}^{n}
\end{array}\right)
$$

we obtain an expression for $\mathbf{T}^{n}$ which depends explicitly on $n$ and does not require $n$ matrix multiplications to evaluate.

\section{The Matched Ellipsoid}

Now consider the matricies

$$
\mathbf{f}=\left(\begin{array}{rr}
\beta_{1} & -\alpha_{1} \\
-\alpha_{1} & \gamma_{1}
\end{array}\right), \quad \mathbf{g}=\left(\begin{array}{rr}
\beta_{2} & -\alpha_{2} \\
-\alpha_{2} & \gamma_{2}
\end{array}\right), \quad \mathbf{D}=\left(\begin{array}{ll}
\mathbf{f} & \mathbf{0} \\
\mathbf{0} & \mathbf{g}
\end{array}\right)
$$

where $\alpha_{1}, \beta_{1}, \gamma_{1}, \alpha_{2}, \beta_{2}, \gamma_{2}$ are the Courant-Snyder parameters defined. above. It follows from (90-92) that

$$
\mathbf{A f A}^{\dagger}=\mathbf{f}, \quad \mathbf{B g B}^{\dagger}=\mathbf{g}, \quad \mathbf{U D U}^{\dagger}=\mathbf{D}
$$

and if we let

$$
\mathbf{E}=\mathbf{R} \mathbf{D} \mathbf{R}^{\dagger},
$$

we have

$$
\mathbf{T E} \mathbf{T}^{\dagger}=\mathbf{R U} \mathbf{R}^{-1}\left(\mathbf{R} \mathbf{D} \mathbf{R}^{\dagger}\right) \mathbf{R}^{-1 \dagger} \mathbf{U}^{\dagger} \mathbf{R}^{\dagger}=\mathbf{R} \mathbf{U D} \mathbf{U}^{\dagger} \mathbf{R}^{\dagger}=\mathbf{R} \mathbf{D} \mathbf{R}^{\dagger}=\mathbf{E} .
$$

Thus, any ellipsoid defined by the matrix $\mathbf{E}=\mathbf{R} \mathbf{D} \mathbf{R}^{\dagger}$ is transformed into the same ellipsoid on each turn around a machine and is said to be matched to the machine lattice. For the remainder of these notes we will use $\mathbf{E}$ to denote the matched ellipsoid matrix. 


\section{Courant-Snyder Invariants}

After passage through $n$ periods of a periodic lattice, a beam particle whose initial coordinates were $x_{0}, x_{0}^{\prime}, y_{0}, y_{0}^{\prime}$ has coordinates $x, x^{\prime}, y, y^{\prime}$ given by

$$
\mathbf{Z}=\mathbf{T}^{n} \mathbf{Z}_{0}
$$

where

$$
\begin{gathered}
\mathbf{Z}_{0}=\left(\begin{array}{c}
\mathbf{X}_{0} \\
\mathbf{Y}_{0}
\end{array}\right), \quad \mathbf{X}_{0}=\left(\begin{array}{c}
x_{0} \\
x_{0}^{\prime}
\end{array}\right), \quad \mathbf{Y}_{0}=\left(\begin{array}{l}
y_{0} \\
y_{0}^{\prime}
\end{array}\right), \\
\mathbf{Z}=\left(\begin{array}{c}
\mathbf{X} \\
\mathbf{Y}
\end{array}\right), \quad \mathbf{X}=\left(\begin{array}{l}
\boldsymbol{x} \\
x^{\prime}
\end{array}\right), \quad \mathbf{Y}=\left(\begin{array}{l}
y \\
y^{\prime}
\end{array}\right)
\end{gathered}
$$

and

$$
\mathbf{T}^{n}=\mathbf{R} \mathbf{U}^{n} \mathbf{R}^{-1}
$$

Defining

$$
\widehat{\mathbf{Z}}=\mathbf{R}^{-1} \mathbf{Z}, \quad \widehat{\mathbf{Z}}_{0}=\mathbf{R}^{-1} \mathbf{Z}_{0}
$$

we then have

$$
\widehat{\mathbf{Z}}=\mathbf{R}^{-1} \mathbf{Z}=\mathbf{R}^{-1}\left(\mathbf{R} \mathbf{U}^{n} \mathbf{R}^{-1}\right) \mathbf{Z}_{0}=\mathbf{U}^{n} \widehat{\mathbf{Z}}_{0}
$$

Then writing

$$
\widehat{\mathbf{Z}}=\left(\begin{array}{c}
\widehat{\mathbf{X}} \\
\widehat{\mathbf{Y}}
\end{array}\right), \quad \widehat{\mathbf{Z}}_{0}=\left(\begin{array}{c}
\widehat{\mathbf{X}}_{0} \\
\widehat{\mathbf{Y}}_{0}
\end{array}\right),
$$

where $\widehat{\mathbf{X}}, \widehat{\mathbf{Y}}, \widehat{\mathbf{X}}_{0}, \widehat{\mathbf{Y}}_{0}$ are two-dimensional vectors, equation (105) becomes

$$
\widehat{\mathbf{X}}=\mathbf{A}^{n} \widehat{\mathbf{X}}_{0}, \quad \widehat{\mathbf{Y}}=\mathbf{B}^{n} \widehat{\mathbf{Y}}_{0}
$$

and it follows from (97) that

$$
\widehat{\mathbf{X}}^{\dagger} \mathbf{f}^{-1} \widehat{\mathbf{X}}=\widehat{\mathbf{X}}_{0}^{\dagger} \mathbf{f}^{-1} \widehat{\mathbf{X}}_{0}=\epsilon_{1}
$$

and

$$
\widehat{\mathbf{Y}}^{\dagger} \mathbf{g}^{-1} \widehat{\mathbf{Y}}=\widehat{\mathbf{Y}}_{0}^{\dagger} \mathbf{g}^{-1} \widehat{\mathbf{Y}}_{0}=\epsilon_{2}
$$

These are the Courant-Snyder Invariants of the motion. Now since

$$
\mathbf{D}^{-1}=\left(\begin{array}{ll}
\mathbf{f}^{-1} & \mathbf{0} \\
\mathbf{0} & \mathbf{g}^{-1}
\end{array}\right)
$$


it follows that

$$
\widehat{\mathbf{Z}}^{\dagger} \mathbf{D}^{-1} \widehat{\mathbf{Z}}=\widehat{\mathbf{Z}}_{0}^{\dagger} \mathbf{D}^{-1} \widehat{\mathbf{Z}}_{0}=\epsilon_{1}+\epsilon_{2}
$$

But we also have

$$
\epsilon=\mathbf{Z}^{\dagger} \mathbf{E}^{-1} \mathbf{Z}=\widehat{\mathbf{Z}}^{\dagger} \mathbf{D}^{-1} \widehat{\mathbf{Z}}
$$

and therefore

$$
\epsilon_{1}+\epsilon_{2}=\epsilon
$$

\section{Range of Motion}

Constraints on the range of positions and angles given by equations (100-103) follow from the Courant-Snyder invariants and from the projections of the matched ellipsoid onto the $x, x^{\prime}$ and $y, y^{\prime}$ planes. The matched ellipsoid is obtained by substituting equations (62) and (96) into (98). Thus

$$
\mathbf{E}=\left(\begin{array}{ll}
\mathbf{F} & \mathbf{C} \\
\mathbf{C}^{\dagger} & \mathbf{G}
\end{array}\right)=\left(\begin{array}{cc}
d \mathbf{I} & \overline{\mathbf{W}} \\
-\mathbf{W} & d \mathbf{I}
\end{array}\right)\left(\begin{array}{ll}
\mathbf{f} & \mathbf{0} \\
\mathbf{0} & \mathbf{g}
\end{array}\right)\left(\begin{array}{cc}
d \mathbf{I} & -\mathbf{W}^{\dagger} \\
\overline{\mathbf{W}}^{\dagger} & d \mathbf{I}
\end{array}\right)
$$

and therefore

$$
\mathbf{F}=d^{2} \mathbf{f}+\overline{\mathbf{W}} \mathbf{g} \overline{\mathbf{W}}^{\dagger}, \quad \mathbf{G}=d^{2} \mathbf{g}+\mathbf{W} \mathbf{f} \mathbf{W}^{\dagger}
$$

Then writing

$$
\mathbf{Z}=\left(\begin{array}{c}
\mathbf{X} \\
\mathbf{Y}
\end{array}\right), \quad \mathbf{X}=\left(\begin{array}{l}
x \\
x^{\prime}
\end{array}\right), \quad \mathbf{Y}=\left(\begin{array}{l}
y \\
y^{\prime}
\end{array}\right)
$$

and using the results of section 4, we obtain the projections of the matched ellipsoid onto the $x, x^{\prime}$ and $y, y^{\prime}$ planes,

$$
\mathbf{X}^{\dagger} \mathbf{F}^{-1} \mathbf{X} \leq \epsilon, \quad \mathbf{Y}^{\dagger} \mathbf{G}^{-1} \mathbf{Y} \leq \epsilon .
$$

The positions and angles in the two planes are constrained to remain inside these ellipses. Further constraints are imposed by the Courant-Snyder invariants. Writing the first of equations (104) as

$$
\mathbf{Z}=\mathbf{R} \widehat{\mathbf{Z}}
$$

and using (62), (102) and (106) we have

$$
\mathbf{X}=d \widehat{\mathbf{X}}+\overline{\mathbf{W}} \widehat{\mathbf{Y}}, \quad \mathbf{Y}=-\mathbf{W} \widehat{\mathbf{X}}+d \widehat{\mathbf{Y}}
$$


and therefore

$$
\mathbf{X}=\mathbf{X}_{1}+\mathbf{X}_{2}, \quad \mathbf{Y}=\mathbf{Y}_{1}+\mathbf{Y}_{2}
$$

where

$$
\mathbf{X}_{1}=d \widehat{\mathbf{X}}, \quad \mathbf{X}_{2}=\overline{\mathbf{W}} \widehat{\mathbf{Y}}, \quad \mathbf{Y}_{1}=-\mathbf{W} \widehat{\mathbf{X}}, \quad \mathbf{Y}_{2}=d \hat{\mathbf{Y}} .
$$

Now if $d \neq 0$ and $|\mathbf{W}| \neq 0$, the matricies $d^{2} \mathbf{f}, \overline{\mathbf{W}} \mathbf{g} \overline{\mathbf{W}}^{\dagger}, \mathbf{W f} \mathbf{W}^{\dagger}, d^{2} \mathbf{g}$ all have inverses and are all positive definite, and we have

$$
\begin{gathered}
\mathbf{X}_{1}^{\dagger}\left(d^{2} \mathbf{f}\right)^{-1} \mathbf{X}_{1}=\widehat{\mathbf{X}}^{\dagger} \mathbf{f}^{-1} \widehat{\mathbf{X}}=\epsilon_{1}, \\
\mathbf{X}_{2}^{\dagger}\left(\overline{\mathbf{W}} \mathbf{g} \overline{\mathbf{W}}^{\dagger}\right)^{-1} \mathbf{X}_{2}=\widehat{\mathbf{Y}}^{\dagger} \mathbf{g}^{-1} \widehat{\mathbf{Y}}=\epsilon_{2}, \\
\mathbf{Y}_{1}^{\dagger}\left(\mathbf{W} \mathbf{f} \mathbf{W}^{\dagger}\right)^{-1} \mathbf{Y}_{1}=\widehat{\mathbf{X}}^{\dagger} \mathbf{f}^{-1} \widehat{\mathbf{X}}=\epsilon_{1}, \\
\mathbf{Y}_{2}^{\dagger}\left(d^{2} \mathbf{g}\right)^{-1} \mathbf{Y}_{2}=\widehat{\mathbf{Y}}^{\dagger} \mathbf{g}^{-1} \widehat{\mathbf{Y}}=\epsilon_{2} .
\end{gathered}
$$

Thus $\mathbf{X}_{1}, \mathbf{X}_{2}, \mathbf{Y}_{1}, \mathbf{Y}_{2}$ are each constrained to lie on an ellipse. The maximum possible values of $x, x^{\prime}, y, y^{\prime}$ are given by the diagonal elements of the ellipse matricies. Thus

$$
\begin{array}{r}
|x| \leq \sqrt{d^{2} f_{11} \epsilon_{1}}+\sqrt{\left(\overline{\mathbf{W}} \mathbf{g} \overline{\mathbf{W}}^{\dagger}\right)_{11} \epsilon_{2}} \\
\left|x^{\prime}\right| \leq \sqrt{d^{2} f_{22} \epsilon_{1}}+\sqrt{\left(\overline{\mathbf{W}} \mathbf{g} \overline{\mathbf{W}}^{\dagger}\right)_{22} \epsilon_{2}} \\
|\boldsymbol{y}| \leq \sqrt{\left.(\mathbf{W f} \mathbf{f})^{\dagger}\right)_{11} \epsilon_{1}}+\sqrt{d^{2} g_{11} \epsilon_{2}} \\
\left|\boldsymbol{y}^{\prime}\right| \leq \sqrt{\left(\mathbf{W} \mathbf{f} \mathbf{W}^{\dagger}\right)_{22} \epsilon_{1}}+\sqrt{d^{2} g_{22} \epsilon_{2}}
\end{array}
$$

where

$$
\begin{aligned}
& f_{11}=\beta_{1}, \quad f_{22}=\gamma_{1}, \quad g_{11}=\beta_{2}, \quad g_{22}=\gamma_{2} \\
& \left(\mathbf{W} \mathbf{f} \mathbf{W}^{\dagger}\right)_{11}=\beta_{1} W_{11}^{2}-2 \alpha_{1} W_{11} W_{12}+\gamma_{1} W_{12}^{2} \\
& \left(\mathbf{W} \mathbf{f} \mathbf{W}^{\dagger}\right)_{22}=\beta_{1} W_{21}^{2}-2 \alpha_{1} W_{21} W_{22}+\gamma_{1} W_{22}^{2} \\
& \left(\overline{\mathbf{W}} \mathbf{g} \overline{\mathbf{W}}^{\dagger}\right)_{11}=\beta_{2} W_{22}^{2}+2 \alpha_{2} W_{22} W_{12}+\gamma_{2} W_{12}^{2} \\
& \left(\overline{\mathbf{W}} \mathbf{g} \overline{\mathbf{W}}^{\dagger}\right)_{22}=\beta_{2} W_{21}^{2}+2 \alpha_{2} W_{21} W_{11}+\gamma_{2} W_{11}^{2}
\end{aligned}
$$




\section{Normal Modes of Oscillation}

Equations (120-125) show that the positions and angles $x, x^{\prime}, y, y^{\prime}$ are given by the superposition of two modes of oscillation which we shall label 1 an 2. In mode 1 we have $\epsilon_{1} \neq 0$ and $\epsilon_{2}=0$, and it follows from (122-125) that $\mathbf{X}_{2}=\mathbf{Y}_{2}=\mathbf{0}$ and therefore $\mathbf{X}=\mathbf{X}_{1}$ and $\mathbf{Y}=\mathbf{Y}_{1}$. Similarly, in mode 2 we have $\epsilon_{2} \neq 0$ and $\epsilon_{1}=0$, and it follows that $\mathbf{X}=\mathbf{X}_{2}$ and $\mathbf{Y}=\mathbf{Y}_{2}$.

Thus, for each mode of oscillation, the motion in each plane is constrained to lie on a single ellipse. If $\epsilon_{1}$ and $\epsilon_{2}$ are both nonzero, then both modes of oscillation are present and the motion in each plane is characterized by the superposition of two ellipses. This characterization of the motion in terms of two ellipses was first derived by Ripken [7, 8].

Now using (107) in (121), and (91) in (94), we have

$$
\begin{gathered}
\mathbf{X}_{1}=d \widehat{\mathbf{X}}=d \mathbf{A}^{n} \widehat{\mathbf{X}}_{0}, \quad \mathbf{X}_{2}=\overline{\mathbf{W}} \widehat{\mathbf{Y}}=\overline{\mathbf{W}} \mathbf{B}^{n} \widehat{\mathbf{Y}}_{0} \\
\mathbf{Y}_{1}=-\mathbf{W} \widehat{\mathbf{X}}=-\mathbf{W A}^{n} \widehat{\mathbf{X}}_{0}, \quad \mathbf{Y}_{2}=d \widehat{\mathbf{Y}}=d \mathbf{B}^{n} \widehat{\mathbf{Y}}_{0}
\end{gathered}
$$

where

$$
\mathbf{A}^{n}=\left(\begin{array}{cc}
\cos n \psi_{1}+\alpha_{1} \sin n \psi_{1} & \beta_{1} \sin n \psi_{1} \\
-\gamma_{1} \sin n \psi_{1} & \cos n \psi_{1}-\alpha_{1} \sin n \psi_{1}
\end{array}\right)
$$

and

$$
\mathbf{B}^{n}=\left(\begin{array}{cc}
\cos n \psi_{2}+\alpha_{2} \sin n \psi_{2} & \beta_{2} \sin n \psi_{2} \\
-\gamma_{2} \sin n \psi_{2} & \cos n \psi_{2}-\alpha_{2} \sin n \psi_{2}
\end{array}\right)
$$

Thus the tunes, $Q_{1}$ and $Q_{2}$, associated with modes 1 and 2 are given by

$$
\psi_{1}=2 \pi Q_{1}, \quad \psi_{2}=2 \pi Q_{2} .
$$

Using

$$
\mathbf{R}^{-1}=\left(\begin{array}{cc}
d \mathbf{I} & -\overline{\mathbf{W}} \\
\mathbf{W} & d \mathbf{I}
\end{array}\right)
$$

in the second of equations (104) we can obtain the vectors $\widehat{\mathbf{X}}_{0}$ and $\widehat{\mathbf{Y}}_{0}$ appearing in (135-136) in terms of $X_{0}$ and $Y_{0}$. Thus

$$
\widehat{\mathbf{X}}_{0}=d \mathbf{X}_{0}-\overline{\mathbf{W}} \mathbf{Y}_{0}, \quad \widehat{\mathbf{Y}}_{0}=\mathbf{W} \mathbf{X}_{0}+d \mathbf{Y}_{0}
$$

and it follows that for mode 1 oscillations $\left(\epsilon_{2}=0\right)$ we must have

$$
\widehat{\mathbf{Y}}_{0}=\mathbf{0}=\mathbf{W X}_{0}+d \mathbf{Y}_{0}
$$


and for mode 2 oscillations $\left(\epsilon_{1}=0\right)$

$$
\widehat{\mathbf{X}}_{0}=0=d \mathbf{X}_{0}-\overline{\mathbf{W}} \mathbf{Y}_{0} \text {. }
$$

These equations give the conditions the initial positions and angles must satisfy in order to excite mode 1 or mode 2 oscillations.

\section{Minimization of $\epsilon$ Subject to Constraints}

In our analysis of injection in the next sections we will need the values of the positions and angles $x_{0}, x_{0}^{\prime}, y_{0}, y_{0}^{\prime}$ which, subject to certain constraints, minimize $\epsilon=\mathbf{Z}_{0}^{\dagger} \mathbf{E}^{-1} \mathbf{Z}_{0}$. Let us first consider the case in which the $n$th component, $Z_{n}$, of $Z_{0}$ is fixed at some value $Z$. We wish to find the other components of $\mathbf{Z}_{0}$ for which

$$
\epsilon=\mathbf{Z}_{0}^{\dagger} \mathbf{E}^{-1} \mathbf{Z}_{0}=F\left(\mathbf{Z}_{0}\right)
$$

is smallest. If the minimum exists we have

$$
\frac{\partial F}{\partial Z_{i}}=2\left(\mathrm{E}^{-1} \mathbf{Z}_{0}\right)_{i}=0, \quad i \neq n
$$

and therefore

$$
\mathbf{E}^{-1} \mathbf{Z}_{0}=\lambda \mathbf{u}, \quad \mathbf{Z}_{0}=\lambda \mathbf{E u}, \quad Z=Z_{n}=\lambda E_{n n}, \quad Z_{i}=Z E_{i n} / E_{n n}
$$

where $\mathbf{u}$ is the unit vector with all but the $n$th component equal to zero-i.e. $u_{i}=\delta_{i n}$. The minimum value of $F\left(\mathbf{Z}_{0}\right)$ is then

$$
\epsilon=\lambda^{2} \mathbf{u}^{\dagger} \mathbf{E u}=\lambda^{2} E_{n n}=Z^{2} / E_{n n}
$$

and the corresponding components of $\mathbf{Z}_{0}$ are given by the last of equations (146).

Now consider the case in which the first and third components of $\mathbf{Z}_{0}$ are fixed at values $x_{0}$ and $y_{0}$. Then we have

$$
\mathbf{E}^{-1} \mathbf{Z}_{0}=\lambda_{1} \mathbf{u}_{1}+\lambda_{3} \mathbf{u}_{3}, \quad \mathbf{z}_{0}=\lambda_{1} \mathbf{E} \mathbf{u}_{1}+\lambda_{3} \mathbf{E} \mathbf{u}_{3}
$$

where $\mathbf{u}_{n}$ is the unit vector with all but the $n$th component equal to zero. Solving (148) for $\lambda_{1}$ and $\lambda_{3}$ we find

$$
\lambda_{1}=\frac{x_{0} E_{33}-y_{0} E_{13}}{E_{11} E_{33}-E_{13}^{2}}, \quad \lambda_{3}=\frac{y_{0} E_{11}-x_{0} E_{13}}{E_{11} E_{33}-E_{13}^{2}} .
$$


The values of the second and fourth components of $\mathbf{Z}_{0}$ for which $\epsilon$ is smallest are then

$$
Z_{2}=\lambda_{1} E_{21}+\lambda_{3} E_{23}, \quad Z_{4}=\lambda_{1} E_{41}+\lambda_{3} E_{43}
$$

and

$$
\epsilon=\lambda_{1}^{2} E_{11}+\lambda_{3}^{2} E_{33}+2 \lambda_{1} \lambda_{3} E_{13} .
$$

For the case in which $y_{0}=0$ we have

$$
\begin{gathered}
Z_{2}=\left(\frac{E_{21} E_{33}-E_{23} E_{13}}{E_{11} E_{33}-E_{13}^{2}}\right) x_{0}, \quad Z_{4}=\left(\frac{E_{41} E_{33}-E_{43} E_{13}}{E_{11} E_{33}-E_{13}^{2}}\right) x_{0} \\
\epsilon=\frac{x_{0}^{2} E_{33}}{E_{11} E_{33}-E_{13}^{2}} .
\end{gathered}
$$

\section{Booster Injection}

Let us now apply the results of the previous sections to the injection of heavy ions into the Booster. The heavy ion beam enters the Booster at the exit of the electrostatic inflector located in the C3 straight section. We assume that the beam emittance is small compared to the acceptance of the Booster and define the initial beam ellipsoid to be the smallest ellipsoid which contains the incoming beam distribution. Thus, if we let

$$
\xi_{0}=\left(\begin{array}{c}
\mathbf{U}_{0} \\
\mathbf{V}_{0}
\end{array}\right), \quad \mathbf{U}_{0}=\left(\begin{array}{c}
u_{0} \\
u_{0}^{\prime}
\end{array}\right), \quad \mathbf{V}_{0}=\left(\begin{array}{c}
v_{0} \\
v_{0}^{\prime}
\end{array}\right)
$$

and

$$
\mathbf{z}_{0}=\left(\begin{array}{c}
\mathbf{X}_{0} \\
\mathbf{Y}_{0}
\end{array}\right), \quad \mathbf{X}_{0}=\left(\begin{array}{c}
x_{0} \\
x_{0}^{\prime}
\end{array}\right), \quad \mathbf{Y}_{0}=\left(\begin{array}{c}
y_{0} \\
y_{0}^{\prime}
\end{array}\right)
$$

where $u_{0}, u_{0}^{\prime}, v_{0}, v_{0}^{\prime}$ are the initial positions and angles (with respect to the equilibrium orbit) of any particle in the incoming beam distribution, and $x_{0}, x_{0}^{\prime}, y_{0}, y_{0}^{\prime}$ are the initial positions and angles (also with respect to the equilibrium orbit) of the beam ellipsoid center, then the initial beam ellipsoid is defined by

$$
\left(\xi_{0}-\mathbf{Z}_{0}\right)^{\dagger} \mathbf{E}_{0}^{-1}\left(\xi_{0}-\mathbf{Z}_{0}\right) \leq \epsilon_{b}
$$


where $\epsilon_{b}$ specifies the emittance. On the $n$th pass by the inflector exit, the positions and angles $x, x^{\prime}, y, y^{\prime}$ of the center of the beam ellipsoid are given by

$$
\mathbf{Z}=\mathbf{T}^{n} \mathbf{Z}_{0}
$$

where

$$
\mathbf{Z}=\left(\begin{array}{c}
\mathbf{X} \\
\mathbf{Y}
\end{array}\right), \quad \mathbf{X}=\left(\begin{array}{c}
x \\
x^{\prime}
\end{array}\right), \quad \mathbf{Y}=\left(\begin{array}{l}
y \\
y^{\prime}
\end{array}\right),
$$

and $\mathbf{T}$ is the transfer matrix for one turn around the Booster starting at the inflector exit. Similarly, the positions and angles of a beam particle on the $n$th pass by the inflector are given by

$$
\boldsymbol{\xi}=\mathbf{T}^{n} \xi_{0},
$$

where

$$
\xi=\left(\begin{array}{c}
\mathbf{U} \\
\mathbf{V}
\end{array}\right), \quad \mathbf{U}=\left(\begin{array}{l}
u \\
u^{\prime}
\end{array}\right), \quad \mathbf{V}=\left(\begin{array}{c}
v \\
v^{\prime}
\end{array}\right)
$$

The beam ellipsoid on the $n$th pass by the inflector is therefore given by

$$
(\xi-\mathbf{Z})^{\dagger} \mathbf{E}_{n}^{-1}(\xi-\mathbf{Z}) \leq \epsilon_{b},
$$

where

$$
\mathbf{E}_{n}=\mathbf{T}^{n} \mathbf{E}_{0} \mathbf{T}^{\dagger n}
$$

and $\mathrm{Z}$ is given by (157). Equations (157) and (162) give the complete turn-by-turn evolution of the beam ellipsoid as it goes around the Booster.

\section{Computer Code}

To study the turn-by-turn evolution of the beam ellipsoid after injection into the Booster, a computer program, CINJ.FOR, has been written to calculate the necessary matricies and parameters. The program requires the Courant-Snyder parameters at the inflector exit, at each quadrupole, and at each skew quadrupole. These are obtained by the MAD code for the case of a bare Booster which contains only the lattice dipoles and quadrupoles and has zero currents in the auxiliary windings on the quadrupoles. (The auxiliary windings on all quadrupoles at horizontal beta maximums are connected in series as are those on all quadrupoles at vertical beta maximums and the currents, $I_{h}$ and $I_{v}$, in the two series 
strings can be adjusted to obtain whatever tunes one desires.) The user of the program specifies the desired values for the uncoupled-i.e. with zero current in the skew quadrupoles-horizontal and vertical tunes, $Q_{x}$ and $Q_{y}$, and the program then calculates the currents, $I_{h}$ and $I_{v}$, required to give these tunes for a particle with magnetic rigidity equal to that of a 1 $\mathrm{GeV} / \mathrm{c}$ Proton. The user then specifies the currents in the skew quadrupoles located in straight sections $1,2,7$ and 8 of each superperiod. We shall consider only the case in which all skew quads are excited with the same current $I_{s}$. Given the the skew quad currents and the bare lattice parameters, and having calculated the currents required to give the desired uncoupled tunes, the program then calculates the one-turn transfer matrix, $\mathbf{T}$, and the matricies, $\mathbf{R}$ and $\mathbf{U}$, which reduce it to block-diagonal form. Once these are obtained, it calculates the Courant-Snyder parameters, given by (86-89), and the matched ellipsoid matricies $\mathbf{D}$ and $\mathbf{E}$ given by (96-98). The user then specifies the initial horizontal and vertical positions, $x_{0}$ and $y_{0}$, of the center of the incoming beam ellipsoid and the program calculates the initial angles, $x_{0}^{\prime}$ and $y_{0}^{\prime}$, which minimize $\epsilon=\mathbf{Z}_{0}^{\dagger} \mathbf{E}^{-1} \mathbf{Z}_{0}$. These are given by equations (149-150). The program then calculates the Courant-Snyder invariants, $\epsilon_{1}$ and $\epsilon_{2}$, and the four ellipses (122-125) associated with the motion of the beam ellipsoid center. The projections (117) of the matched ellipsoid $\mathbf{Z}^{\dagger} \mathbf{E}^{-1} \mathbf{Z}=\epsilon$ onto the $x, x^{\prime}$ and $y, y^{\prime}$ planes are also calculated. The initial positions and angles of the beam ellipsoid center are then substituted into equation (157) and the positions and angles on subsequent turns around the machine are calculated. The user must also specify the initial beam ellipsoid matrix $\mathbf{E}_{0}$ which the program uses in equation (162) to calculate $\mathbf{E}_{n}$ on subsequent turns around the machine. The half-widths of the beam ellipsoid in the two planes on the $n$th pass by the inflector are then given by

$$
W_{x}=\sqrt{\epsilon_{b}\left(\mathbf{E}_{n}\right)_{11}}, \quad W_{y}=\sqrt{\epsilon_{b}\left(\mathbf{E}_{n}\right)_{33}}
$$

where $\epsilon_{b}$ specifies the beam emittance.

\section{Injection Parameters to be Optimized}

If the position of the equilibrium orbit at the exit of the inflector is held fixed during injection, then on subsequent passes by the inflector, the injected beam will eventually hit the inflector and be lost. The orbit must 
therefore be moved away from the inflector at appropriate times during injection in order to keep the injected beam in the machine. Eventually the orbit has to be moved so far from the inflector that none of the incoming beam enters the acceptance region of the machine, and at this point the injection process comes to an end. Clearly, then, the longer one can wait before having to move the orbit away from the inflector, the more beam one can inject into the machine and keep. Optimization of the injection process therefore consists of finding incoming beam parameters $x_{0}, x_{0}^{\prime}, y_{0}, y_{0}^{\prime}$, and $\mathbf{E}_{0}$, and machine parameters $Q_{x}, Q_{y}$, and $I_{s}$ which keep the center of the beam ellipsoid away from the inflector for as long as possible and at the same time keep the shape of the ellipsoid under control so that none of its edges scrape on the inflector. For given initial positions, $x_{0}$ and $y_{0}$, of the beam ellipsoid center, the optimum angles, $x_{0}^{\prime}$ and $y_{0}^{\prime}$, are those which minimize $\epsilon=\mathbf{Z}_{0}^{\dagger} \mathbf{E}^{-1} \mathbf{Z}_{0}$. We shall assume that the initial beam ellipsoid has no correlation between the horizontal and vertical planes. The matrix $\mathbf{E}_{0}$ is then of the form

$$
\mathbf{E}_{0}=\left(\begin{array}{cc}
\mathbf{F}_{0} & \mathbf{0} \\
\mathbf{0} & \mathbf{G}_{0}
\end{array}\right)=\left(\begin{array}{cc}
\epsilon_{x} \mathbf{A}_{x} / \epsilon_{b} & \mathbf{0} \\
\mathbf{0} & \epsilon_{y} \mathbf{A}_{y} / \epsilon_{b}
\end{array}\right)
$$

where

$$
\mathbf{A}_{x}=\left(\begin{array}{cc}
\beta_{x} & -\alpha_{x} \\
-\alpha_{x} & \gamma_{x}
\end{array}\right), \quad \mathbf{A}_{y}=\left(\begin{array}{cc}
\beta_{y} & -\alpha_{y} \\
-\alpha_{y} & \gamma_{y}
\end{array}\right)
$$

and

$$
\beta_{x} \gamma_{x}-\alpha_{x}^{2}=1, \quad \beta_{y} \gamma_{y}-\alpha_{y}^{2}=1, \quad \sqrt{\epsilon_{x} \epsilon_{y}}=\epsilon_{b} .
$$

The projections of the initial beam ellipsoid (156) on the horizontal and vertical planes are then

$$
\left(\mathbf{U}_{0}-\mathbf{X}_{0}\right)^{\dagger} \mathbf{A}_{x}^{-1}\left(\mathbf{U}_{0}-\mathbf{X}_{0}\right) \leq \epsilon_{x}, \quad\left(\mathbf{V}_{0}-\mathbf{Y}_{0}\right)^{\dagger} \mathbf{A}_{y}^{-1}\left(\mathbf{V}_{0}-\mathbf{Y}_{0}\right) \leq \epsilon_{y}
$$

and we see that $\epsilon_{x}$ and $\epsilon_{y}$ are the horizontal and vertical emittances of the incoming beam. We shall assume that $\epsilon_{x}=\epsilon_{y}$ and that $\alpha_{x}, \beta_{x}, \gamma_{x}, \alpha_{y}, \beta_{y}$, $\gamma_{y}$ are matched to the uncoupled lattice parameters at the inflector exit. With these assumptions we find that, for the situations discussed below, the horizontal and vertical widths (163) of the beam ellipsoid on subsequent turns around the machine vary only slightly from their initial values. 


\section{Tunes and Skew Quadrupole Currents}

Having specified the incoming beam parameters we turn our attention to the machine parameters $Q_{x}, Q_{y}$ and $I_{s}$ and examine their effect on the injection process. Let us first write down approximate expressions for the normal-mode tunes $Q_{1}$ and $Q_{2}$. We know from the perturbation treatment of linear coupling $[9,10]$ that if the uncoupled tunes, $Q_{x}$ and $Q_{y}$, are sufficiently close to each other and the skew quadrupole strength is sufficiently small, then, to first order, the normal-mode tunes are given by

$$
Q_{1}=\frac{1}{2}\left(Q_{x}+Q_{y}\right)+\frac{1}{2} \sqrt{(\Delta Q)^{2}+4 \kappa^{2}}
$$

and

$$
Q_{2}=\frac{1}{2}\left(Q_{x}+Q_{y}\right)-\frac{1}{2} \sqrt{(\Delta Q)^{2}+4 \kappa^{2}}
$$

where

$$
\Delta Q=Q_{y}-Q_{x},
$$

and in our case $\kappa$ is proportional to the skew quadrupole current $I_{s}$. Subtracting (169) from (168) we have

$$
Q_{1}-Q_{2}=\sqrt{(\Delta Q)^{2}+4 \kappa^{2}}
$$

and it follows that

$$
Q_{2}-Q_{1} \leq \Delta Q \leq Q_{1}-Q_{2} .
$$

Thus to obtain particular values for $Q_{1}$ and $Q_{2}$ we first pick some uncoupled tune separation, $\Delta Q$, which satisfies (172) and then adjust the quadrupole currents, $I_{h}$ and $I_{v}$, so that

$$
Q_{x}=\left(Q_{1}+Q_{2}-\Delta Q\right) / 2, \quad Q_{y}=\left(Q_{1}+Q_{2}+\Delta Q\right) / 2 .
$$

With these values for the uncoupled tunes we then adjust the skew quadrupole current to obtain the desired normal-mode tunes.

Now, it has been show in Ref. [11] that for the case in which there is no coupling between the horizontal and vertical planes, one of several possible optimum horizontal tunes is $Q_{x}=4+4 / 5=4.8$. In this case the turn-by-turn motion of the beam ellipsoid center in the horizontal plane is characterized by a single ellipse and the injected beam returns to its initial position after 5 turns around the machine. This situation is depicted in 
Fig. (1) where the beam ellipsoid center has been given initial coordinates $x_{0}=10 \mathrm{~mm}$ and $y_{0}=0$. The ellipse shown is matched to the machine lattice at the inflector exit and the diamonds indicate the positions and angles of the beam ellipsoid center on the $n$th pass by the inflector. When we introduce coupling with the skew quadrupoles, the turn-by-turn motion in the horizontal plane is characterized by the superposition of two ellipses associated with the two normal modes of oscillation as shown in sections 9 and 10. If the coupling is not too strong, then one of the two ellipses will be large and the other one small, and if we choose the normal-mode tune associated with the larger ellipse to be 4.8 , we can, as with the uncoupled case, wait for 5 turns before having to move the equilibrium orbit away from the inflector. But now, by judicious choice of the normal-mode tune associated with the smaller ellipse, one can wait even longer. We illustrate this with some examples.

\section{Examples}

Suppose we adjust $Q_{x}, Q_{y}$, and $I_{3}$ so that the normal-mode tunes associated with the larger and smaller ellipses are $Q_{2}=4+12 / 15=4.8$ and $Q_{1}=4+13 / 15=4.866666$ respectively. Then it will take 15 turns around the machine before the beam ellipsoid center returns to its initial position at the inflector exit. This situation is depicted in Fig. (2a) where the beam ellipsoid center has been given initial coordinates $x_{0}=10 \mathrm{~mm}$ and $y_{0}=0$. The quadrupole currents, $I_{h}$ and $I_{v}$, have been adjusted here so that $Q_{x}=4.823333$ and $Q_{y}=4.843333$, and $I_{s}$ has then been adjusted to give the desired normal-mode tunes. $\left(I_{s}=14.5 \mathrm{Amps}\right.$ for a $1 \mathrm{GeV} / \mathrm{c}$ Proton.) The ellipses labeled 1 and 2 are the normal-mode 1 and 2 ellipses given by (122) and (123), and the ellipse labeled $F$ is the projection of the matched ellipsoid given by the first of equations (117). The diamonds indicate the horizontal positions and angles of the ellipsoid center on the $n$th pass by the inflector. Inspection of the figure shows that the beam ellipsoid center has a horizontal position of at most $4.75 \mathrm{~mm}$ before returning to its initial position at the inflector exit. This is to be compared with the uncoupled situation depicted in Fig. (2b). Here the skew quadrupole current has been set to zero and the quadrupole currents have been adjusted so that $Q_{x}=4+13 / 15$. As before, the beam ellipsoid center has been given initial coordinates $x_{0}=10 \mathrm{~mm}, y_{0}=0$ and it returns to its initial position after 15 turns around the machine. The ellipse shown is 
matched to the uncoupled lattice at the inflector exit and the diamonds, as before, show the positions and angles of the beam ellipsoid center on the $n$th pass by the inflector. In this case we see that the beam ellipsoid center has a horizontal position of at most $9.1 \mathrm{~mm}$ before returning to its initial position. Clearly, one does much better with the coupled situation depicted in Fig. (2a). We note that projection of the matched ellipsoid shown in Fig. (2a) is very nearly the same size and shape as the ellipse matched to the uncoupled lattice shown in Fig. (2b). This is because the strength of the skew quadrupoles is small compared to that of the lattice quadrupoles and we have stayed away from the $Q_{x}+Q_{y}=9$ and $Q_{x}+Q_{y}=10$ resonances. Now, although the initial position, $y_{0}$, of the beam ellipsoid center in the vertical $\left(y, y^{\prime}\right)$ plane is zero, the coupling introduced by the skew quadrupoles produces oscillations of nonzero amplitude in this plane. This is the price we pay for using coupling to extend the time we can wait before having to move the equilibrium orbit away from the inflector. The positions and angles of the beam ellipsoid center in the vertical plane are shown in Fig. (3a) where the ellipse labeled $G$ is the projection of the matched ellipsoid given by the second of equations (117) and the other two ellipses are the normal-mode ellipses given by (124) and (125). Inspection of the normal-mode ellipses shows that the maximum possible value of the vertical position, $y$, of the beam. ellipsoid center is $5.9 \mathrm{~mm}$. The positions in the $x, y$ plane and the projection of the matched ellipsoid onto this plane are shown in Fig. (3b).

For the situation depicted in Figs. (2a) and (3), the uncoupled tune separation is $\Delta Q=.02$. Let us now consider different tune separations while keeping the normal-mode tunes, $Q_{1}$ and $Q_{2}$, the same. As before, the beam ellipsoid center is given initial coordinates $x_{0}=10 \mathrm{~mm}, y_{0}=0$ and we plot the positions and angles in each plane on subsequent turns around the machine. Figures (4-9) show the positions, angles and ellipses for tune separations of $.06, .05, .04, .03, .01$, and 0 respectively. Here we see that as the tune separation decreases toward zero, the larger of the two normal-mode ellipses in the horizontal plane becomes smaller and the smaller of the two ellipses becomes larger. When the uncoupled tune separation reaches zero, the two ellipses are the same size. In the vertical plane the two normal-mode ellipses are the same size and both become larger as the uncoupled tune separation decreases toward zero. This is as expected since we know from the perturbation treatment of linear coupling that the effect of the coupling increases as the uncoupled tune separation 
approaches zero. Table I summarizes the data of Figs. (2-9) and includes data for some additional examples. In each case, the beam ellipsoid center has been given initial coordinates $x_{0}=10 \mathrm{~mm}, y_{0}=0$ and it returns to its initial position after 15 turns around the machine. $x^{*}$ is the horizontal position of the beam ellipsoid center on its closest approach to the inflector before returning to its initial position, and $y^{*}$ is the maximum possible value of the vertical position $y$. The skew quadrupole current, $I_{s}$, is the current (in Amps) required for $1 \mathrm{GeV} / \mathrm{c}$ protons. Comparing all of the examples listed, we see that the beam ellipsoid center stays furthest from the inflector for the case in which $\Delta Q=.02, Q_{2}=4+12 / 15$ and $Q_{1}=4+13 / 15$. Note also that $y^{*}$ decreases as $\Delta Q$ increases.

\begin{tabular}{|l|l|c|r|r|c|c|c|}
\hline \multicolumn{7}{|c|}{ Table I: $15-$ Turn Injection } \\
\hline$Q_{x}$ & $Q_{y}$ & $\Delta Q$ & $I_{s}$ & $Q_{2}-4$ & $Q_{1}-4$ & $\boldsymbol{x}^{*}$ & $\boldsymbol{y}^{*}$ \\
\hline 4.833333 & 4.833333 & $\mathbf{0}$ & $\mathbf{1 5 . 1 0}$ & $12 / 15$ & $13 / 15$ & 5.05 & 6.16 \\
4.828333 & 4.838333 & .01 & $\mathbf{1 5 . 0 0}$ & $12 / 15$ & $13 / 15$ & 4.80 & 6.08 \\
4.823333 & 4.843333 & .02 & 14.50 & $12 / 15$ & $13 / 15$ & 4.75 & 5.85 \\
4.818333 & 4.848333 & .03 & 13.50 & $12 / 15$ & $13 / 15$ & 5.90 & 5.47 \\
4.813333 & 4.853333 & .04 & 12.20 & $12 / 15$ & $13 / 15$ & 6.98 & 4.90 \\
4.808333 & 4.858333 & .05 & 10.00 & $12 / 15$ & $13 / 15$ & 8.13 & 4.03 \\
4.803333 & 4.863333 & .06 & 6.60 & $12 / 15$ & $13 / 15$ & $\mathbf{9 . 2 5}$ & $\mathbf{2 . 6 5}$ \\
\hline 4.867666 & 4.867666 & $\mathbf{0}$ & 30.50 & $12 / 15$ & $14 / 15$ & 6.13 & 6.13 \\
4.857666 & 4.877666 & .02 & $\mathbf{3 0 . 1 0}$ & $12 / 15$ & $14 / 15$ & 5.68 & $\mathbf{6 . 0 4}$ \\
4.847666 & 4.887666 & .04 & 29.10 & $12 / 15$ & $14 / 15$ & 5.23 & 5.81 \\
4.837666 & 4.897666 & .06 & 27.20 & $12 / 15$ & $14 / 15$ & 5.86 & 5.42 \\
4.827266 & 4.907266 & .08 & 24.45 & $12 / 15$ & $14 / 15$ & 6.98 & 4.84 \\
\hline 4.771866 & 4.761866 & -.01 & 14.80 & $11 / 15$ & $12 / 15$ & $\mathbf{5 . 6 7}$ & $\mathbf{6 . 1 4}$ \\
4.776866 & 4.756866 & -.02 & 14.30 & $11 / 15$ & $12 / 15$ & $\mathbf{5 . 2 1}$ & 5.94 \\
4.781866 & 4.751866 & -.03 & 13.40 & $11 / 15$ & $12 / 15$ & 5.88 & 5.57 \\
\hline
\end{tabular}

Let us now consider normal-mode tunes for which 20 turns around the machine are required before the beam ellipsoid center returns to its initial position at the inflector exit. As before, the beam ellipsoid center is given initial coordinates $x_{0}=10 \mathrm{~mm}$ and $y_{0}=0$, and $Q_{x}, Q_{y}$, and $I_{s}$ are adjusted to give the desired normal-mode tunes. The data are summarized in Table II. 


\begin{tabular}{|l|l|c|r|c|c|c|c|}
\hline \multicolumn{10}{|c|}{ Table II: 20-Turn Injection } \\
\hline$Q_{x}$ & $Q_{y}$ & $\Delta Q$ & $I_{s}$ & $Q_{2}-4$ & $Q_{1}-4$ & $x^{*}$ & $y^{*}$ \\
\hline 4.8711 & 4.8811 & .01 & 34.20 & $16 / 20$ & $19 / 20$ & 6.11 & 6.10 \\
4.8661 & 4.8861 & .02 & 34.05 & $16 / 20$ & $19 / 20$ & 5.93 & 6.05 \\
4.8611 & 4.8911 & .03 & 33.70 & $16 / 20$ & $19 / 20$ & 5.97 & 5.97 \\
4.8561 & 4.8961 & .04 & 33.10 & $16 / 20$ & $19 / 20$ & 6.34 & 5.87 \\
\hline 4.825 & 4.825 & 0 & 11.30 & $16 / 20$ & $17 / 20$ & 5.63 & 6.16 \\
4.82 & 4.83 & .01 & 11.10 & $16 / 20$ & $17 / 20$ & 6.02 & 6.03 \\
4.815 & 4.835 & .02 & 10.40 & $16 / 20$ & $17 / 20$ & 7.01 & 5.63 \\
\hline 4.726 & 4.726 & 0 & 33.50 & $13 / 20$ & $16 / 20$ & 5.75 & 6.23 \\
4.731 & 4.721 & -.01 & 33.40 & $13 / 20$ & $16 / 20$ & 5.58 & 6.23 \\
4.736 & 4.716 & -.02 & 33.20 & $13 / 20$ & $16 / 20$ & 5.72 & 6.20 \\
4.741 & 4.711 & -.03 & 32.90 & $13 / 20$ & $16 / 20$ & 6.07 & 6.14 \\
4.746 & 4.706 & -.04 & 32.30 & $13 / 20$ & $16 / 20$ & 6.37 & 6.05 \\
\hline
\end{tabular}

Continuing along these lines we consider normal-mode tunes for which 25 and 30 turns around the machine are required before the beam ellipsoid center returns to its initial position. These data are summarized in Tables III and IV.

\begin{tabular}{|l|l|c|r|c|c|c|c|}
\hline \multicolumn{1}{|c|}{ Table III: 25-Turn Injection } \\
\hline$Q_{x}$ & $Q_{y}$ & $\Delta Q$ & $I_{s}$ & $Q_{2}-4$ & $Q_{1}-4$ & $x^{*}$ & $y^{*}$ \\
\hline 4.820 & 4.820 & 0 & 9.05 & $20 / 25$ & $21 / 25$ & 6.57 & 6.17 \\
4.817 & 4.823 & .006 & 9.00 & $20 / 25$ & $21 / 25$ & 7.07 & 6.09 \\
4.815 & 4.825 & .01 & 8.80 & $20 / 25$ & $21 / 25$ & 7.41 & 5.96 \\
4.810 & 4.830 & .02 & 7.80 & $20 / 25$ & $21 / 25$ & 8.30 & 5.31 \\
\hline 4.78 & 4.78 & 0 & 9.00 & $19 / 25$ & $20 / 25$ & 6.58 & 6.19 \\
4.785 & 4.775 & -.01 & 8.80 & $19 / 25$ & $20 / 25$ & 7.51 & 6.01 \\
\hline 4.76 & 4.76 & 0 & 18.10 & $18 / 25$ & $20 / 25$ & 6.74 & 6.21 \\
4.765 & 4.755 & -.01 & 17.90 & $18 / 25$ & $20 / 25$ & 7.11 & 6.17 \\
\hline 4.741 & 4.741 & 0 & 26.80 & $17 / 25$ & $20 / 25$ & 6.66 & 6.22 \\
4.746 & 4.736 & -.01 & 26.70 & $17 / 25$ & $20 / 25$ & 6.94 & 6.21 \\
4.751 & 4.731 & -.02 & 26.40 & $17 / 25$ & $20 / 25$ & 7.23 & 6.16 \\
\hline
\end{tabular}




\begin{tabular}{|l|l|c|c|c|c|c|c|}
\hline \multicolumn{7}{|c|}{ Table IV: 30-Turn Injection } \\
\hline$Q_{x}$ & $Q_{y}$ & $\Delta Q$ & $I_{s}$ & $Q_{2}-4$ & $Q_{1}-4$ & $x^{*}$ & $y^{*}$ \\
\hline 4.816666 & 4.816666 & 0 & 7.55 & $24 / 30$ & $25 / 30$ & 7.51 & 6.17 \\
4.814166 & 4.819166 & .005 & 7.48 & $24 / 30$ & $25 / 30$ & 7.88 & 6.09 \\
4.811666 & 4.821666 & .01 & 7.23 & $24 / 30$ & $25 / 30$ & 8.25 & 5.87 \\
4.806666 & 4.826666 & .02 & 6.10 & $24 / 30$ & $25 / 30$ & 9.00 & 4.93 \\
\hline 4.783333 & 4.783333 & 0 & 7.50 & $23 / 30$ & $24 / 30$ & 7.52 & 6.19 \\
4.788333 & 4.778333 & -.01 & 7.20 & $23 / 30$ & $24 / 30$ & 8.30 & 5.92 \\
4.793333 & 4.773333 & -.02 & 6.00 & $23 / 30$ & $24 / 30$ & 9.01 & 4.97 \\
\hline 4.718166 & 4.718166 & 0 & 37.1 & $19 / 30$ & $24 / 30$ & 7.51 & 6.24 \\
4.723166 & 4.713166 & -.01 & 37.0 & $19 / 30$ & $24 / 30$ & 7.70 & 6.24 \\
4.728166 & 4.708166 & -.02 & 36.8 & $19 / 30$ & $24 / 30$ & 7.86 & 6.22 \\
\hline
\end{tabular}

Table V lists the entries from Tables I-IV for which the beam ellipsoid center stays furthest from the inflector before returning to its initial position. Here we see that as $n$ (the number of turns required before the beam ellipsoid center returns to its initial position) increases, the horizontal position, $x^{*}$, of the beam ellipsoid center on its closest approach to the inflector increases. Figs. (10) and (11) show plots of the horizontal positions, angles, and ellipses for each entry of Table V.

\begin{tabular}{|l|l|c|r|c|c|c|c|c|}
\hline \multicolumn{10}{|c|}{ Table V } \\
\hline$Q_{x}$ & $Q_{y}$ & $\Delta Q$ & $I_{s}$ & $Q_{2}-4$ & $Q_{1}-4$ & $n$ & $x^{*}$ & $y^{*}$ \\
\hline 4.823333 & 4.843333 & .02 & 14.50 & $12 / 15$ & $13 / 15$ & 15 & 4.75 & 5.85 \\
4.731 & 4.721 & -.01 & 33.40 & $13 / 20$ & $16 / 20$ & 20 & 5.58 & 6.23 \\
4.82 & 4.82 & 0 & 9.05 & $20 / 25$ & $21 / 25$ & 25 & 6.57 & 6.17 \\
4.816666 & 4.816666 & 0 & 7.55 & $24 / 30$ & $25 / 30$ & 30 & 7.51 & 6.17 \\
\hline
\end{tabular}

\section{Guidelines}

We summarize the results of the previous sections with the following guidelines for selecting coupled injection parameters.

1. The initial vertical position, $y_{0}$, of the beam ellipsoid center (with respect to the equilibrium orbit) should be zero. The initial horizontal position, $x_{0}$, (again, with respect to the equilibrium orbit) will vary as the equilibrium orbit collapses away from the inflector. For given values of $x_{0}$ and $y_{0}$, the angles $x_{0}^{\prime}$ and $y_{0}^{\prime}$ should be adjusted to minimize $\epsilon=\mathbf{Z}_{0}^{\dagger} \mathbf{E}^{-1} \mathbf{Z}_{0}$. 
2. The initial beam ellipsoid matrix, $\mathbf{E}_{0}$, should be matched to the uncoupled machine lattice at the inflector exit as discussed in section 14 .

3. The tune associated with the larger of the two normal-mode ellipses in the horizontal plane should be $4+4 / 5=4.8$.

4. The other normal-mode tune should then be chosen so that $n$ turns around the machine are required before the beam ellipsoid center returns to its initial position. We have explored the cases $n=15,20,25$, and 30 . The values of $Q_{x}, Q_{y}$, and $I_{s}$ which give the desired normal-mode tunes for these cases are listed in Tables $\mathrm{I}-\mathrm{V}$ along with the corresponding values of $\boldsymbol{x}^{*}$ and $\boldsymbol{y}^{*}$.

\section{References}

1. T. Roser, Multiturn Injection with Coupling, AGS/AD/Tech. Note No. 354, November 7, 1991.

2. See e.g. J. Powell and B. Crasemann, Quantum Mechanics, Addison-Wesley Publishing Co., p. 477 (1961).

3. E.D. Courant and H.S. Snyder, Annals of Physics 3, 1-48 (1958).

4. L.C. Teng, Concerning n-Dimensional Coupled Motions, NAL Report FN-229, May 3, 1971.

5. D.A. Edwards and L.C. Teng, Parametrization of Linear Coupled Motion in Periodic Systems, IEEE Trans. on Nucl. Sc. 20, 885 (1973).

6. T. Roser, Generalized Treatment of Linear Motion in Two-Dimensional Periodic Systems, University of Michigan Report UM-HE 88-8 (1988).

7. G. Ripken, DESY Report R1-70/04 (1970).

8. F. Willeke and G. Ripken, AIP Conference Proceedings No. 184, pp. 758-819 (1989).

9. G. Guiguard, CERN Report 76-06, March 23, 1976.

10. K. Takikawa, CERN Report ISR-MA/75-34, July 30, 1975.

11. C.J. Gardner, Multiturn Injection of Heavy Ions into the Booster, Booster Tech. Note No. 197, August 14, 1991. 
Fig. 1

UNCOUPLED INJECTION $X, X P$ PLANE

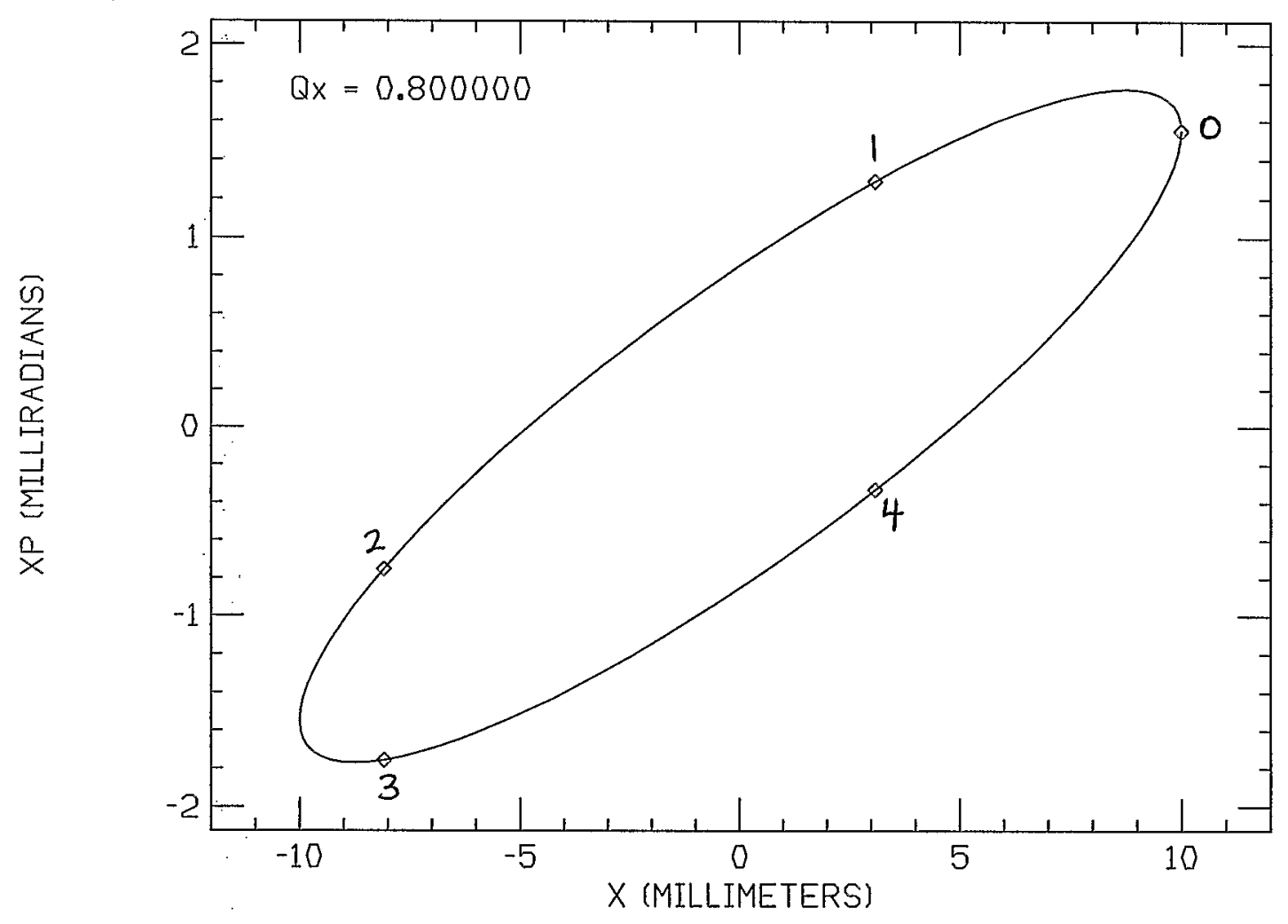


COUPLED INJECTION $X, X P$ PROJECTION

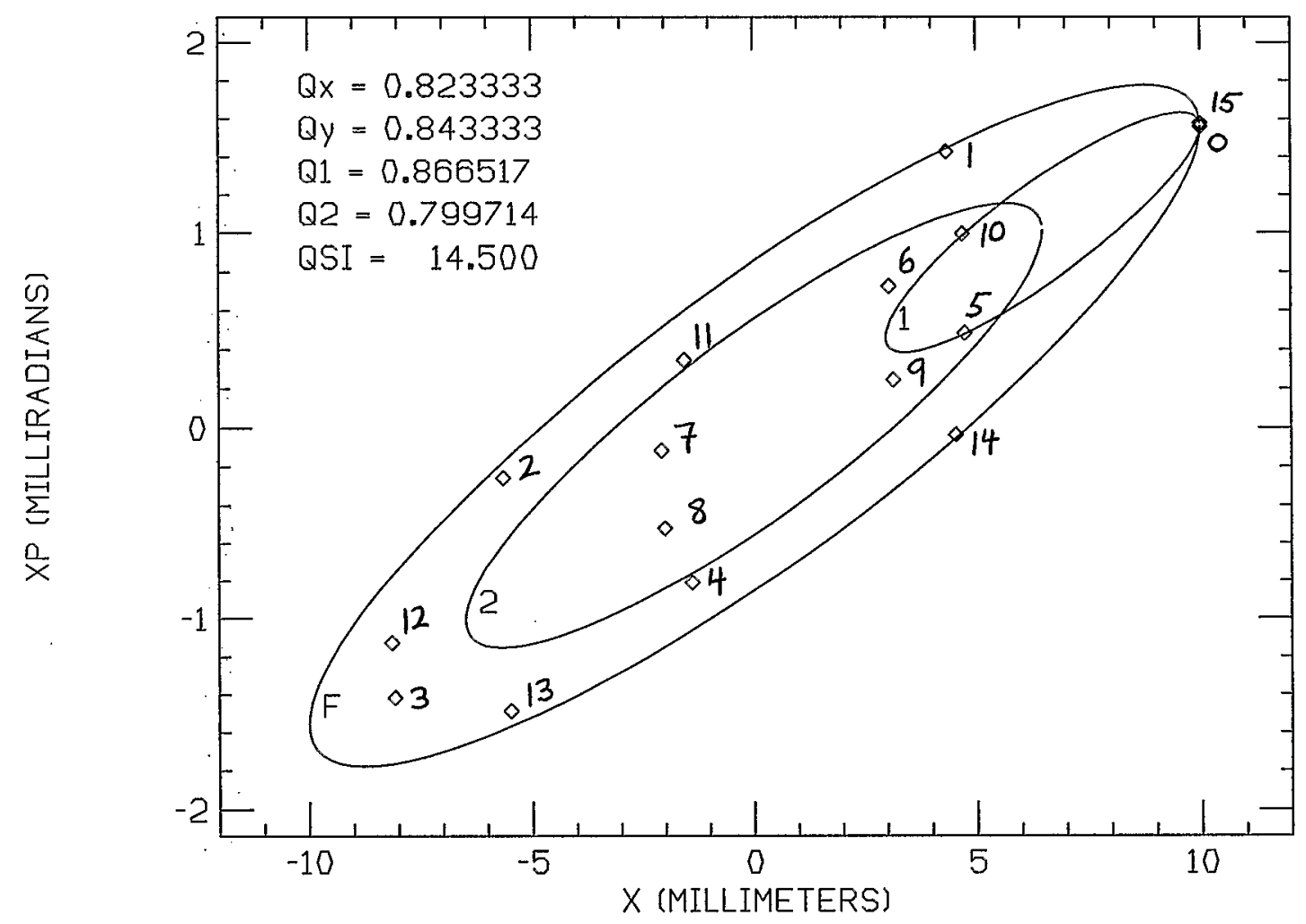

Fig. $2 b$

UNCOUPLED INJECTION $x, X P$ PLANE

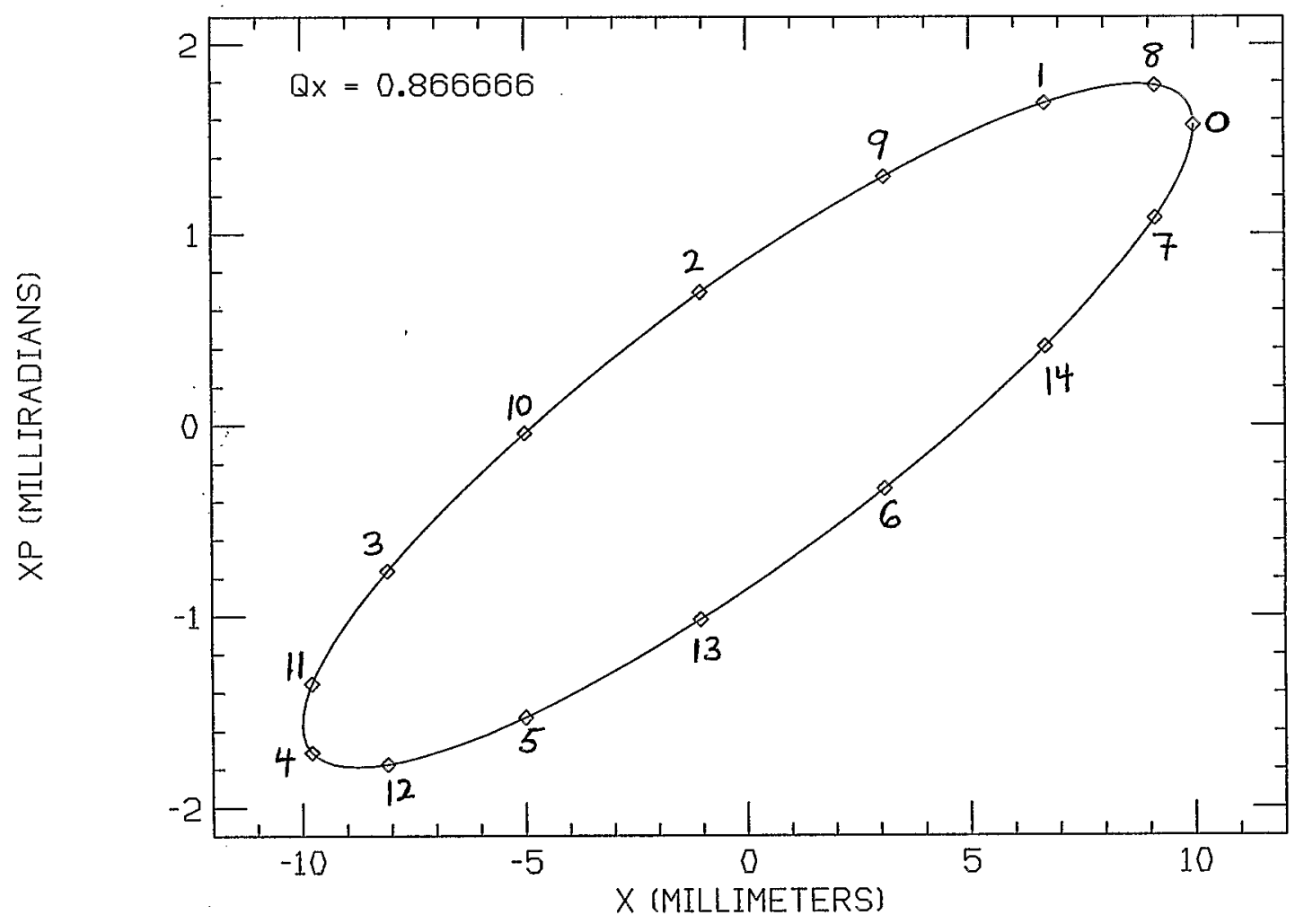


Fig. $3 a$

Y,YP PROJECTION

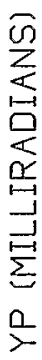

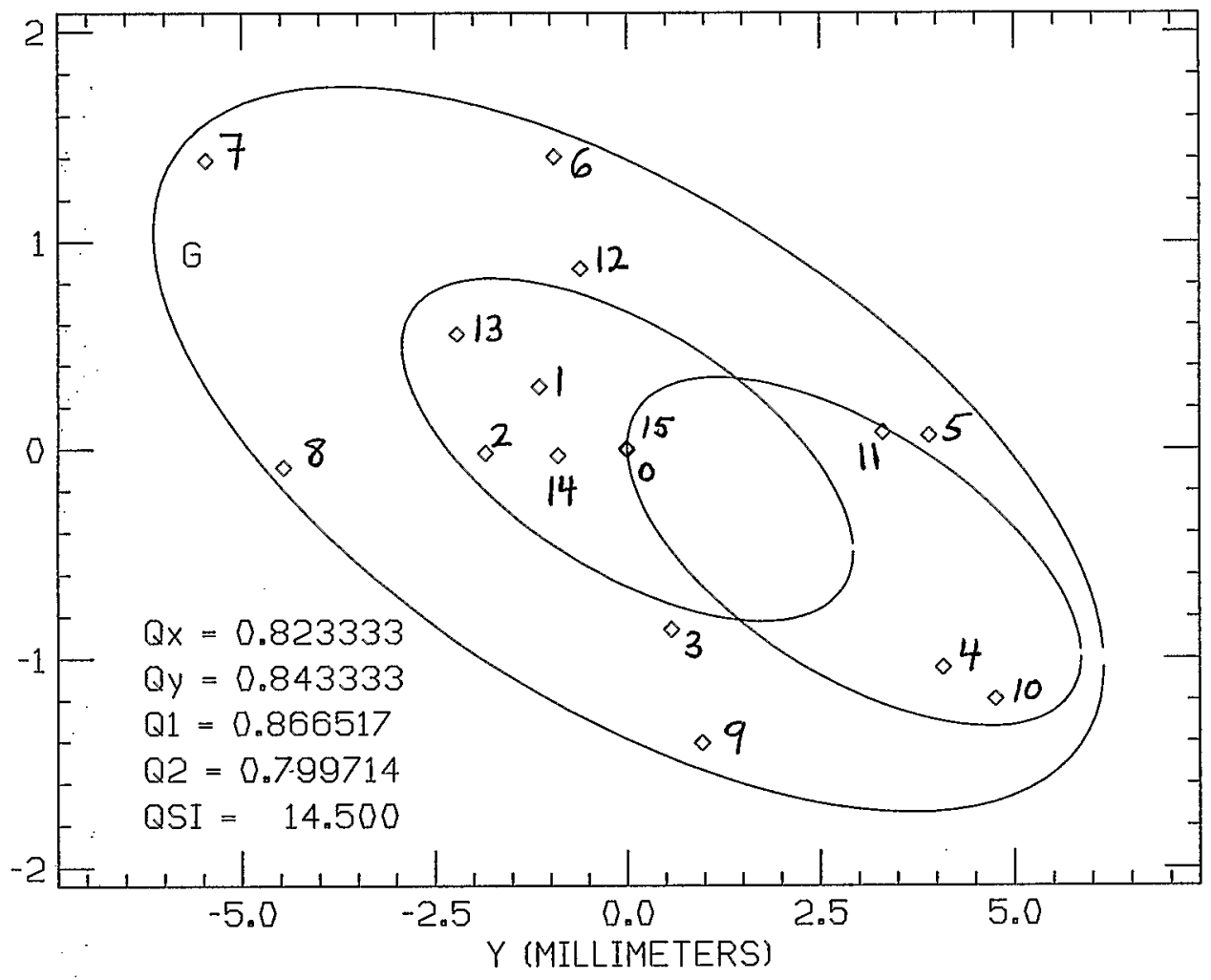

Fig. $3 b$

$X, Y$ PROJECTION

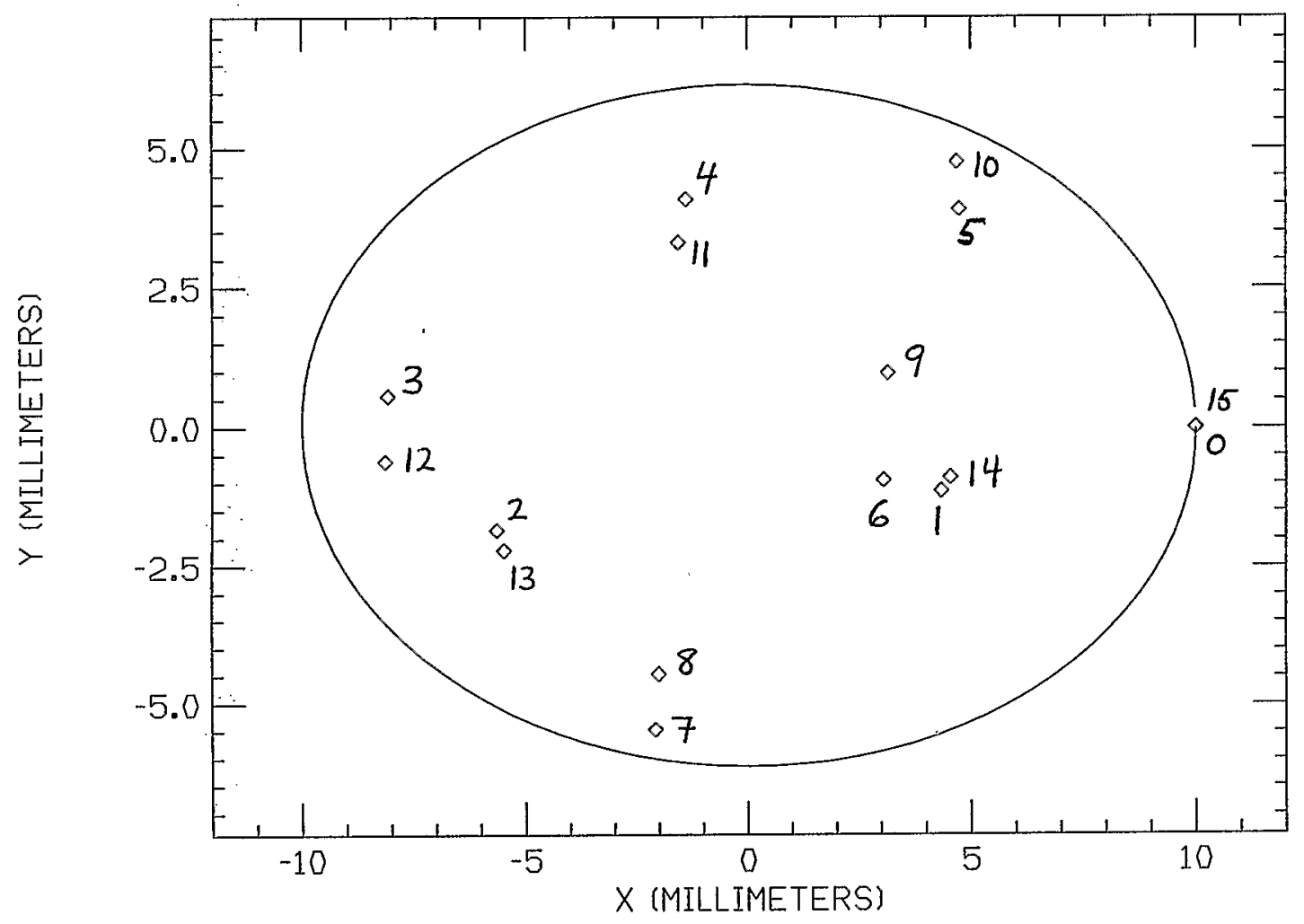


Fig. $4 a$

COUPLED INJECTION $X$, XP PROJECTION

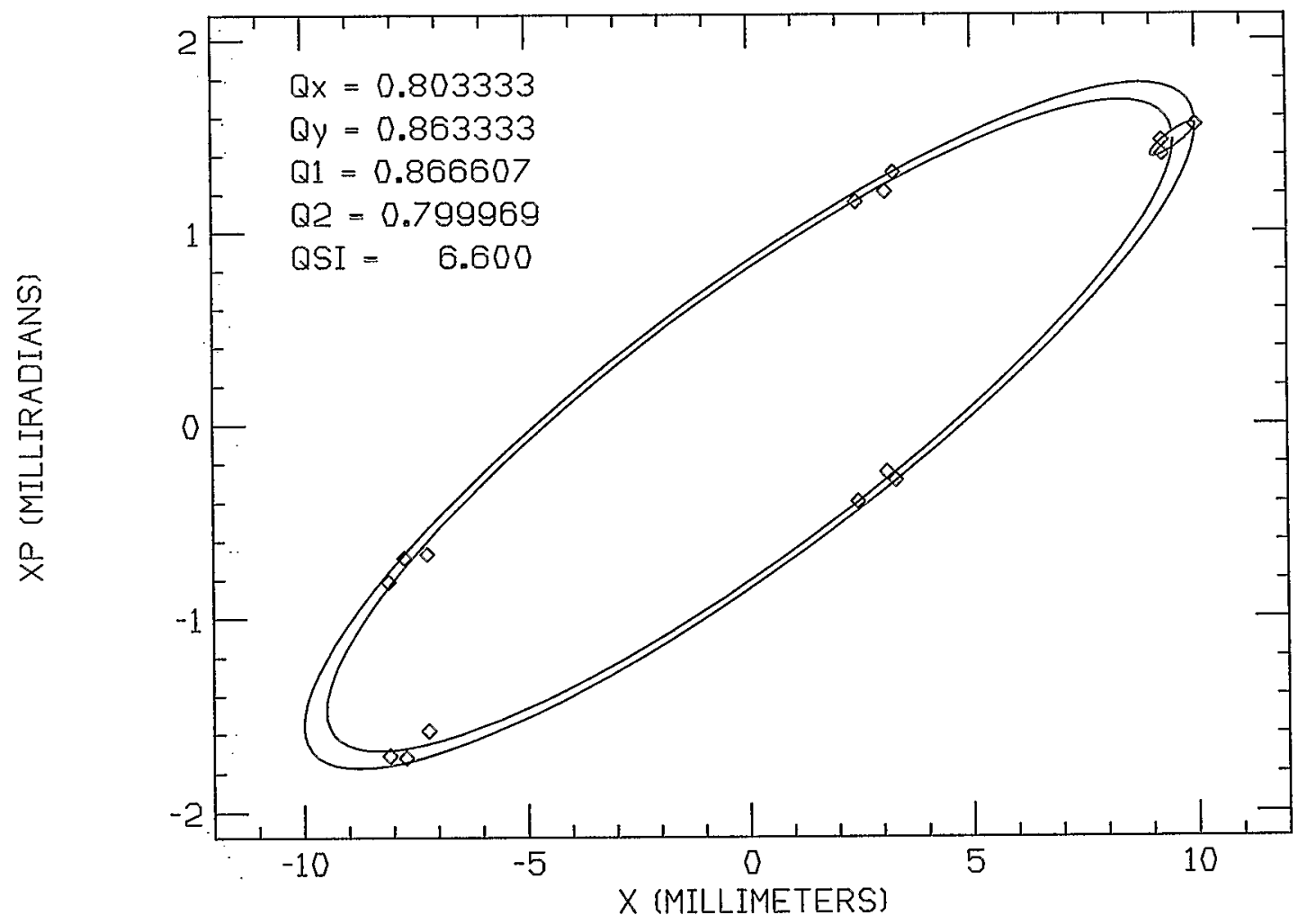

Fig. $4 b$

Y,YP PROJECTION

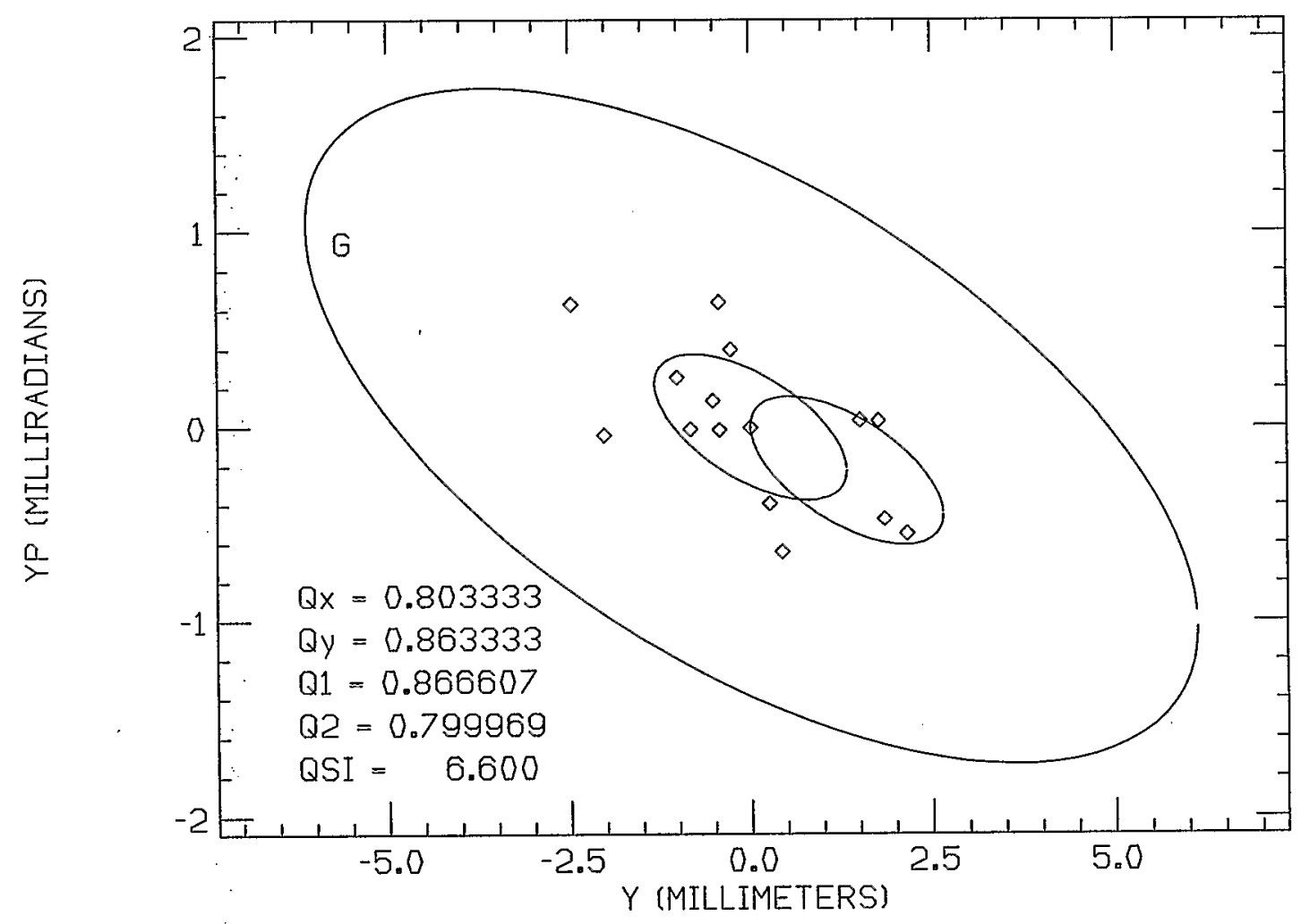


COUPLED INJECTION $X, X P$ PROJECTION

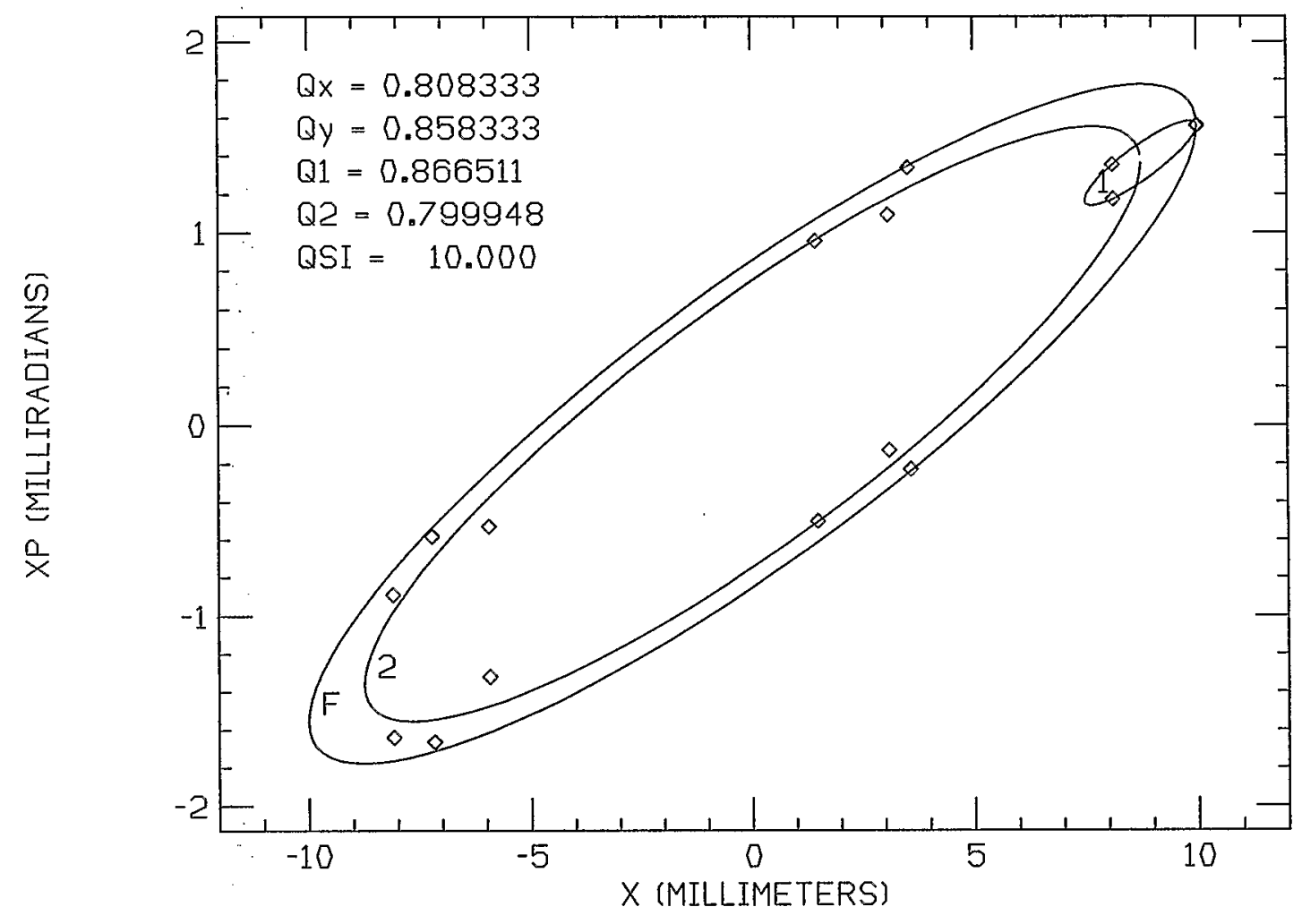

Fig. $5 b$

Y,YP PROJECTION

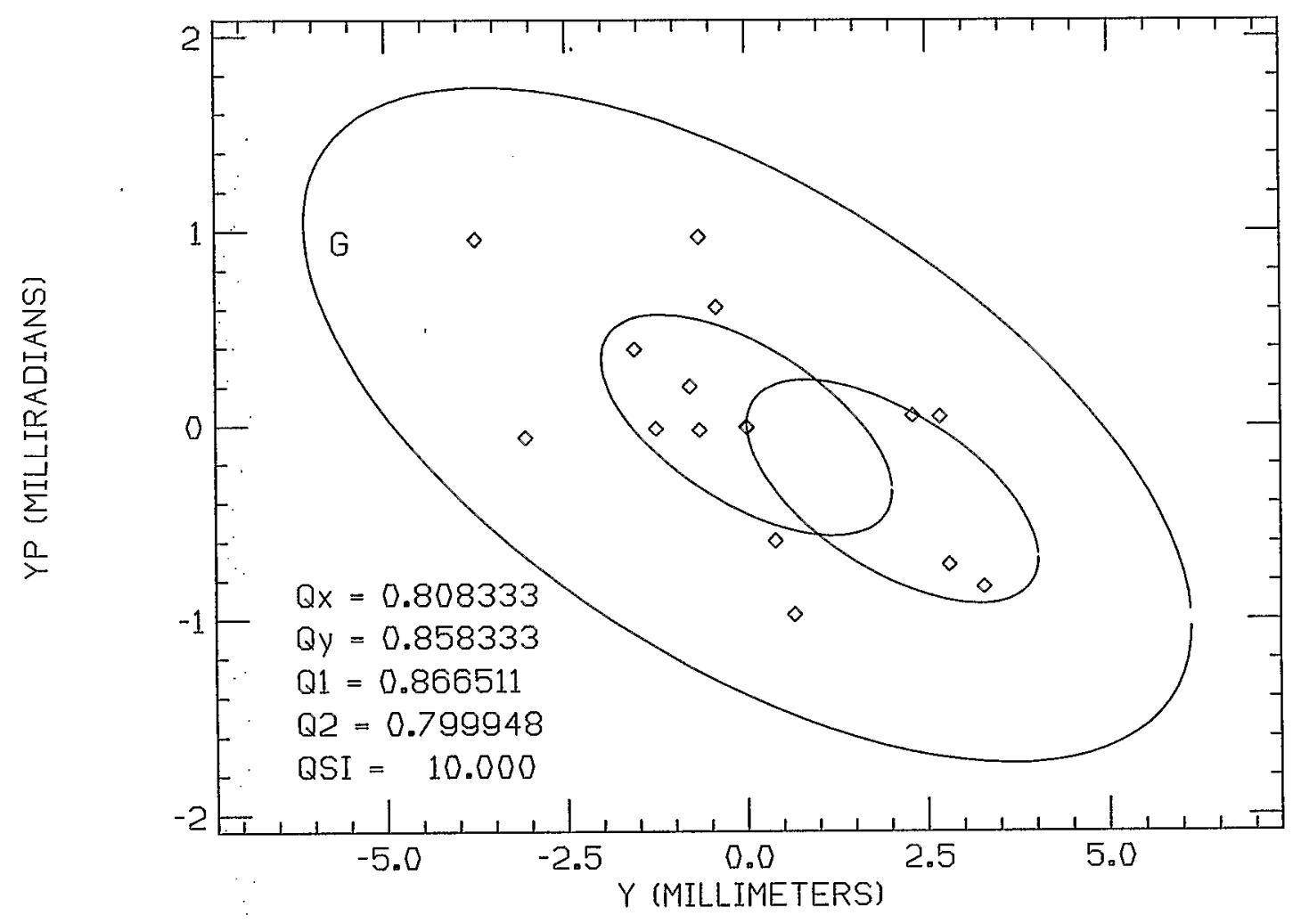




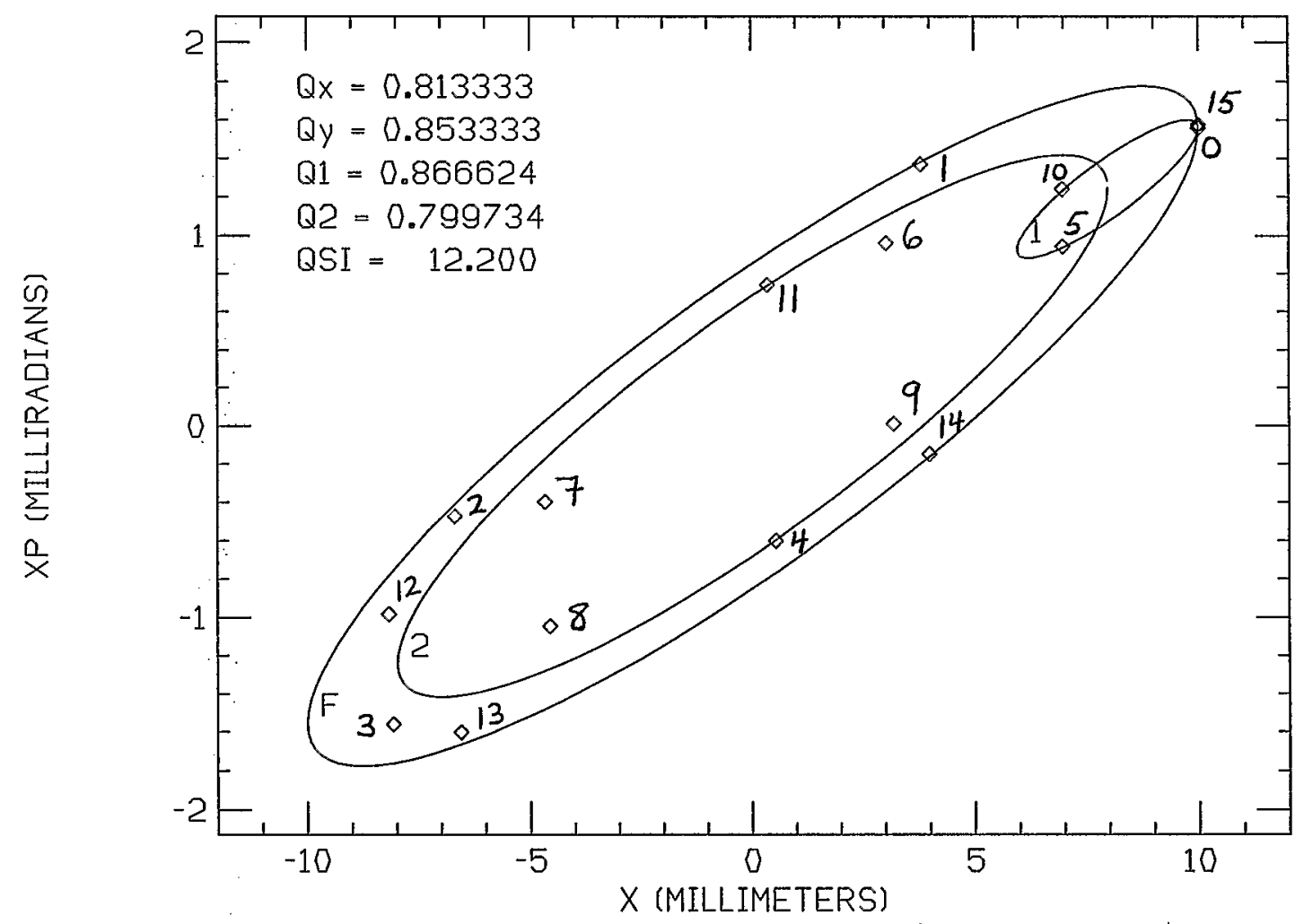

Fig. 6b

Y,YP PROJECTION

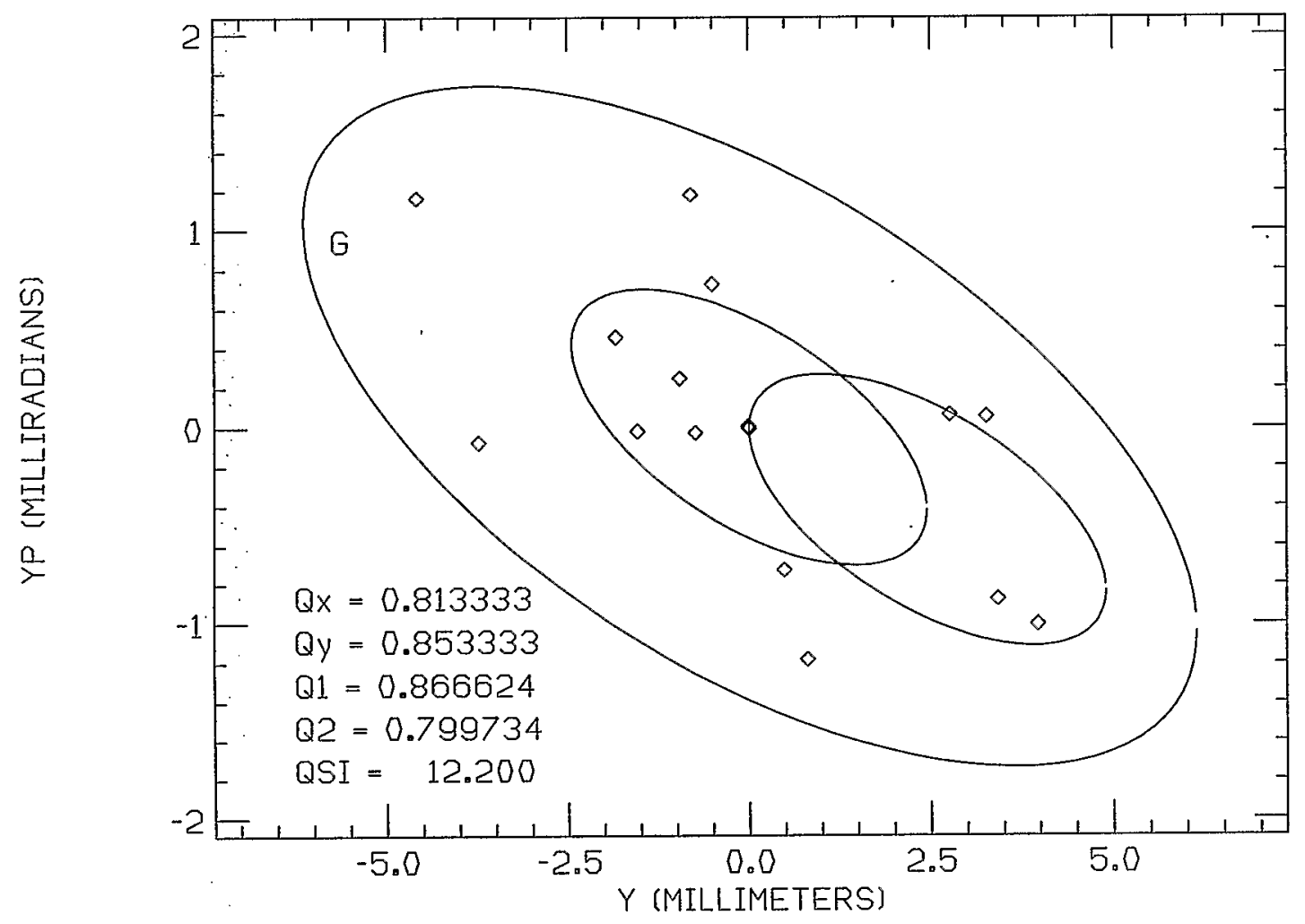




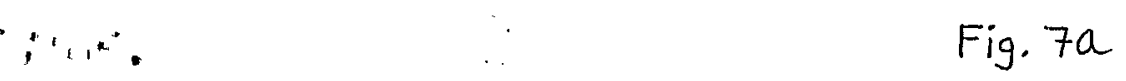

COUPLED INJECTION $X ;$ XP PROJECTION

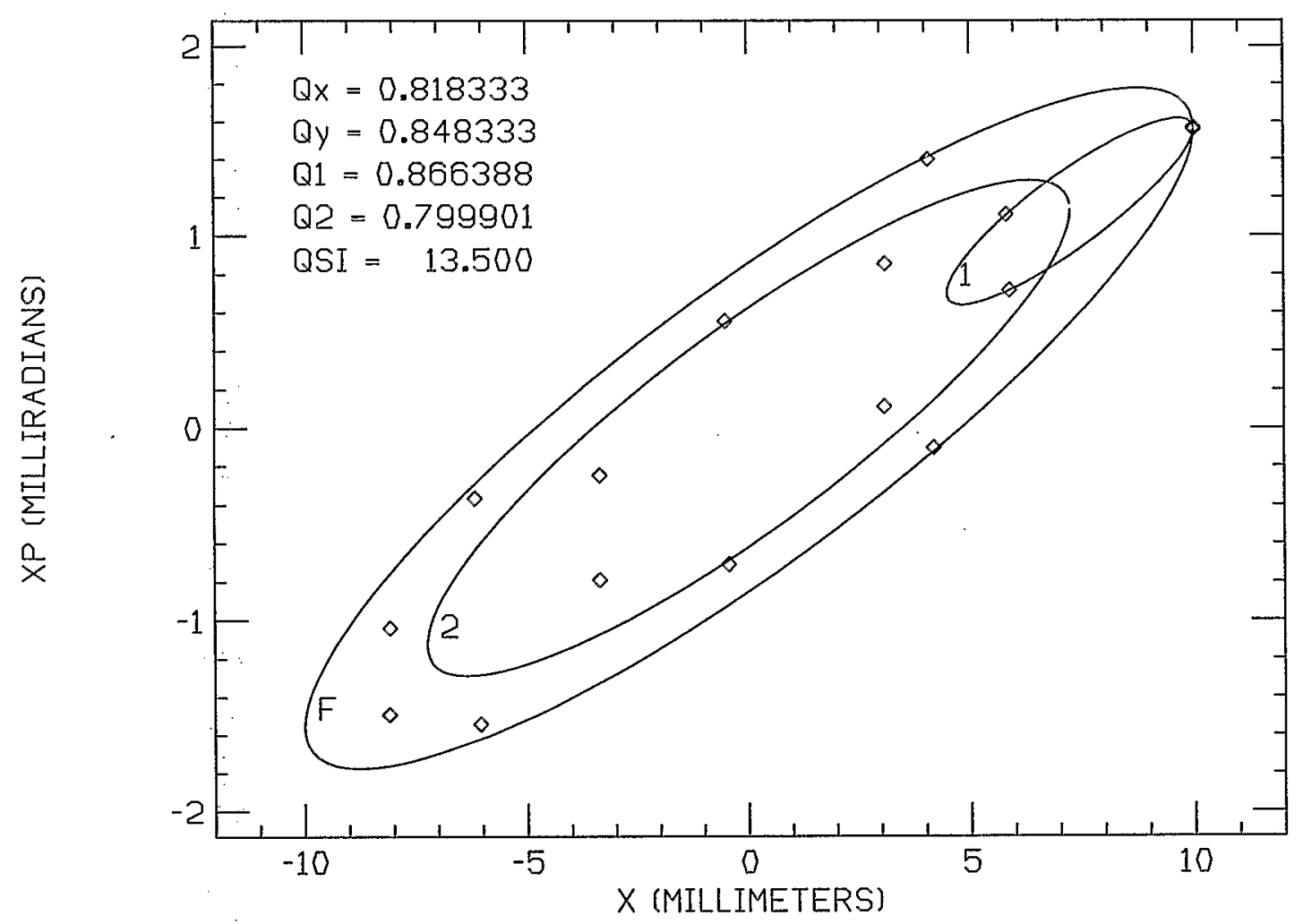

Fig. $7 b$

Y,YP PROJECTION

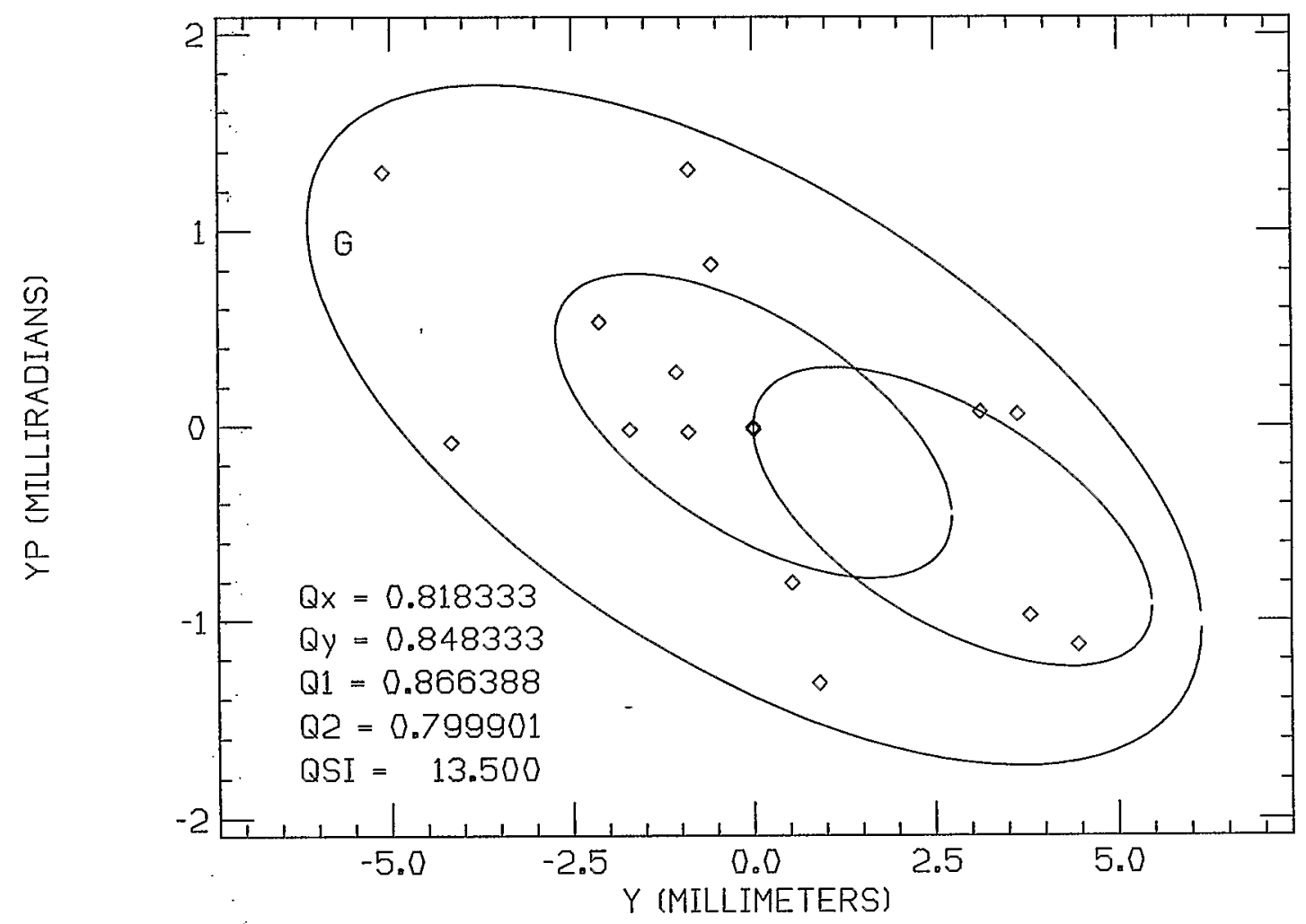


$={ }^{2}+\mathrm{k}^{2}, \quad$ Fig. $8 a$

COUPLED INJECTION X, XP PROJECTION

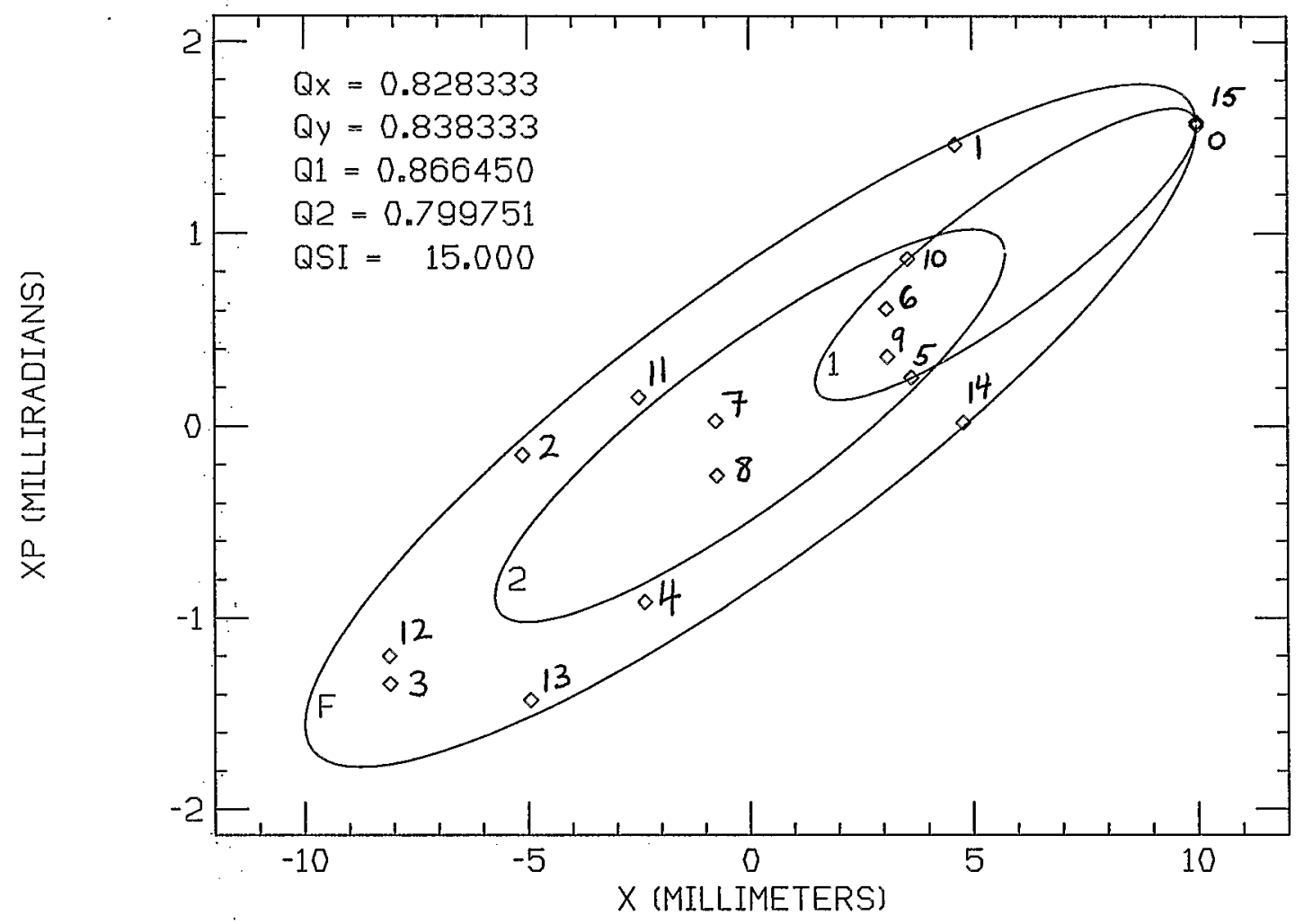

Fig. $8 b$

Y,YP PROJECTION

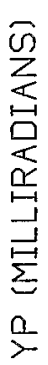

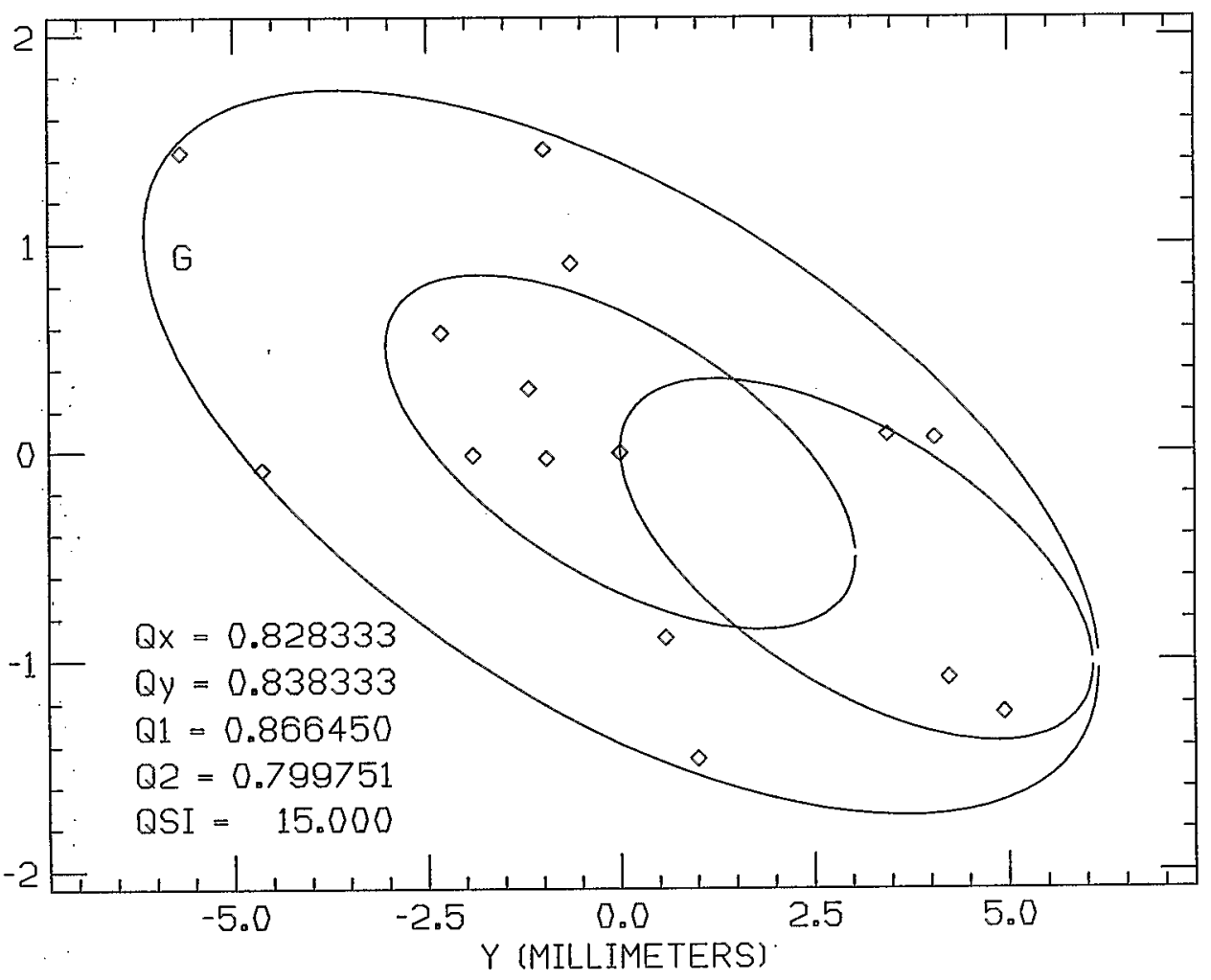




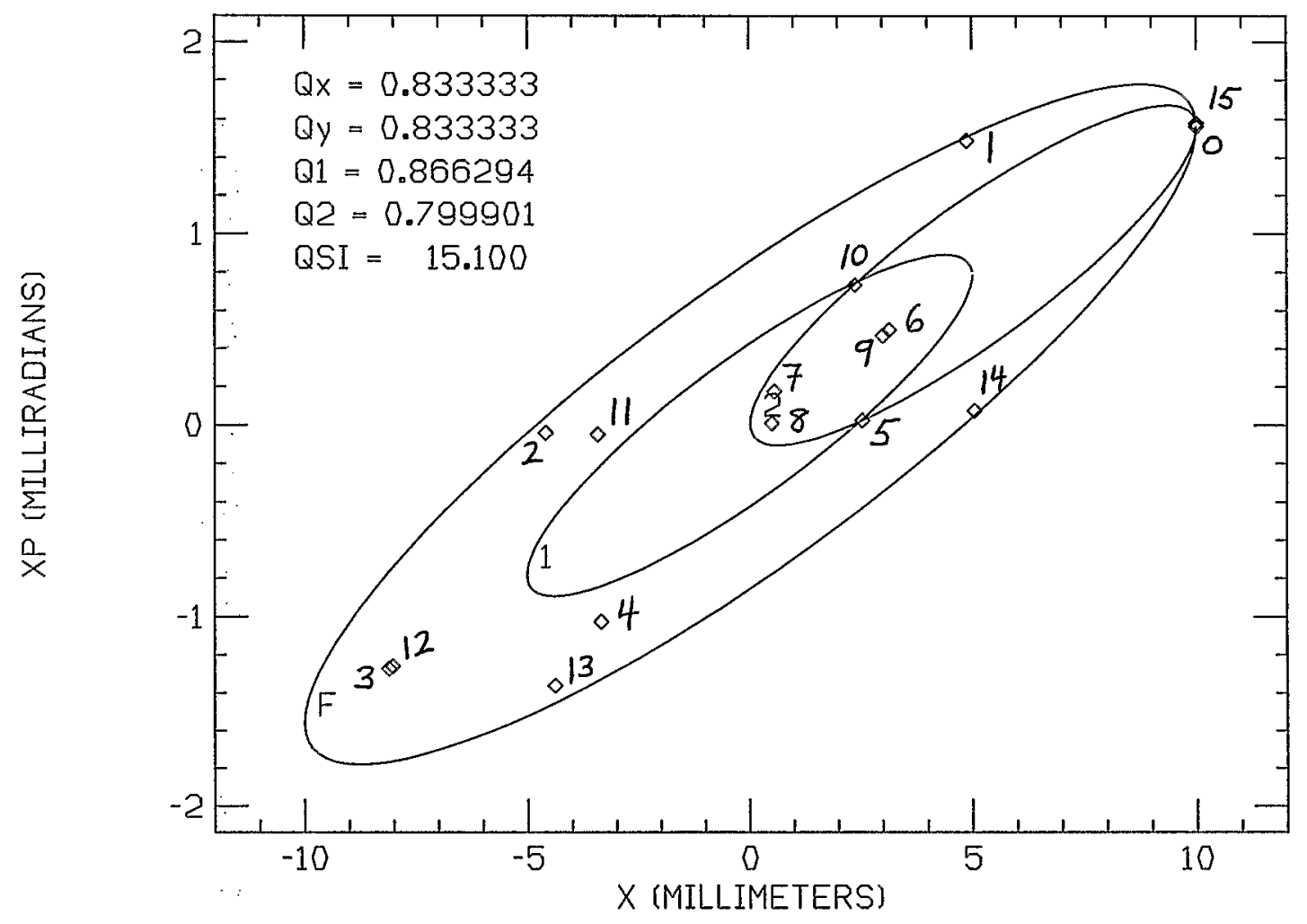

Fig. 96

Y,YP PROJECTION

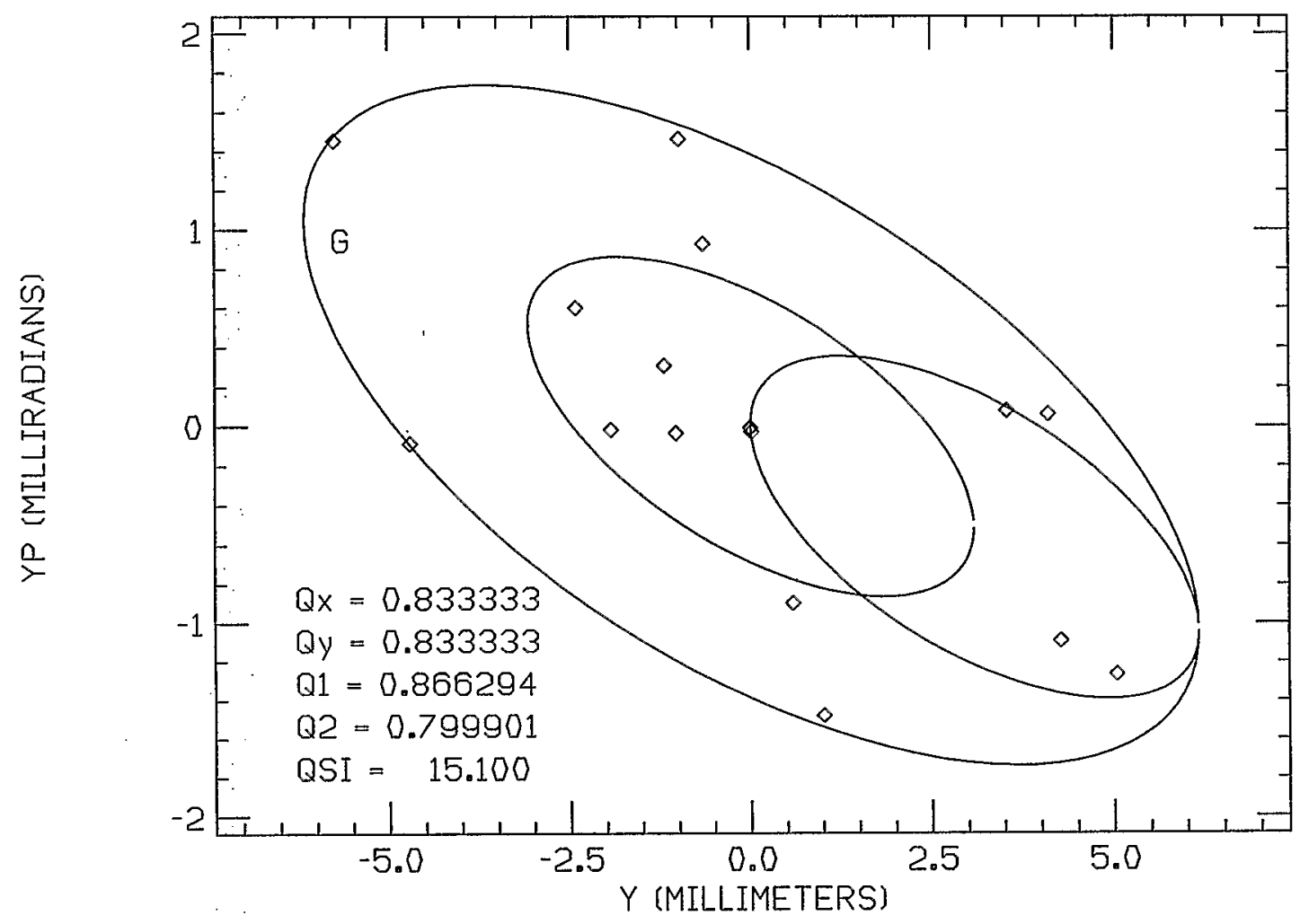

